\title{
APPROXIMATING LONGEST CYCLES IN GRAPHS WITH BOUNDED DEGREES*
}

\author{
GUANTAO $\mathrm{CHEN}^{\dagger}$, ZHICHENG GAO ${ }^{\ddagger}$, XINGXING YU§ ${ }^{\S}$ AND WENAN ZANG
}

\begin{abstract}
Jackson and Wormald conjecture that if $G$ is a 3-connected $n$-vertex graph with maximum degree $d \geq 4$, then $G$ has a cycle of length $\Omega\left(n^{\log _{d-1} 2}\right)$. We show that this conjecture holds when $d-1$ is replaced by $\max \{64,4 d+1\}$. Our proof implies a cubic algorithm for finding such a cycle.
\end{abstract}

Key words. bounded degree, 3-connected components, long cycles, algorithm

AMS subject classifications. $05 \mathrm{C} 38,35 \mathrm{C} 45,05 \mathrm{C} 85$

DOI. $10.1137 / 050633263$

1. Introduction. From the point of view of approximation algorithms, finding a longest cycle in a graph is one of the "harder" NP-hard problems. There is no known polynomial time algorithm which guarantees an approximation ratio better than $n / \operatorname{polylog}(n)$. For graphs with a cycle of length $k$, it is shown in [1] that one can find in polynomial time a cycle of length $\Omega\left((\log k)^{2} / \log \log k\right)$. Gabow [6] showed how to find in polynomial time a cycle of length $\exp (\Omega(\sqrt{\log k / \log \log k}))$ through a given vertex $v$ in a graph that contains a cycle of length $k$ through $v$. Recently, Feder and Motwani [5] obtained a cubic algorithm which, given a graph with maximum degree $d$ and containing a $k$-vertex 3 -cyclable minor, finds a cycle of length $k^{1 /(2 c \log d)}$ for some $c \geq 2$. A consequence of their result improves Gabow's result in certain situations.

Karger, Motwani, and Ramkumar [10] showed that unless $\mathcal{P}=\mathcal{N} \mathcal{P}$ it is impossible to find, in polynomial time, a path of length $n-n^{\epsilon}$ in an $n$-vertex Hamiltonian graph for any $\epsilon<1$. They conjecture that it is as hard even for graphs with bounded degrees. On the other hand, Feder, Motwani, and Subi [4] showed that there is a polynomial time algorithm for finding a cycle of length at least $n^{\left(\log _{3} 2\right) / 2}$ in any 3-connected cubic $n$-vertex graph. They also proposed the problem for 3 -connected graphs with bounded degrees. For a graph $G$, let $\Delta(G)$ denote its maximum degree. Jackson and Wormald [9] proved that every 3 -connected $n$-vertex graph $G$ with $\Delta(G) \leq d$ has a cycle of length at least $\frac{1}{2} n^{\log _{b} 2}+1$, where $b=6 d^{2}$. Recently, Chen, $\mathrm{Xu}$, and $\mathrm{Yu}[3]$ gave a cubic algorithm that, given a 3 -connected $n$-vertex graph $G$ with $\Delta(G) \leq d$, finds a cycle of length at least $n^{\log _{b} 2}$, where $b=2(d-1)^{2}+1$. It was conjectured in 1993 by Jackson and Wormald [9] that for $d \geq 4$ the right value for $b$ should be $d-1$.

*Received by the editors June 8, 2005; accepted for publication (in revised form) April 17, 2006; published electronically October 3, 2006.

http://www.siam.org/journals/sicomp/36-3/63326.html

$\dagger$ Department of Math and Statistics, Georgia State University, Atlanta, GA 30303 and Faculty of Math and Statistics, Huazhong Normal University, Wuhan, China (gchen@gsu.edu). The work of this author was partially supported by NSA grant H98230-04-1-0300 and NSF grant DMS-0500951.

${ }^{\ddagger}$ School of Math and Statistics, Carleton University, Ottawa, Ontario, K1S 5B6, and Center for Combinatorics, LPMC, Nankai University, Tianjin, 300071, China (zgao@math.carleton.ca). The work of this author was partially supported by NSERC and RGC grant HKU7056/04P.

$\S$ School of Mathematics, Georgia Institute of Technology, Atlanta, GA 30332 and Center for Combinatorics, LPMC, Nankai University, Tianjin, 300071, China (yu@math.gatech.edu). The work of this author was partially supported by NSF grant DMS-0245530, NSA grant MDA904-03-1-0052, and RGC grant HKU7056/04P.

【Department of Mathematics, University of Hong Kong, Hong Kong, China (wzang@maths. hku.hk). The work of this author was partially supported by RGC grant HKU7056/04P. 
The main result of this paper shows that this conjecture holds for a linear function $b$ of $d$. (This result appears in the extended abstract [2].)

TheOREM 1.1. Let $n \geq 4$ and $d \geq 4$ be integers. Let $G$ be a 3 -connected graph with $n$ vertices and $\Delta(G) \leq d$. Then $G$ contains a cycle of length at least $\frac{1}{2} n^{\log _{b} 2}+3$, where $b=\max \{64,4 d+1\}$.

For 3-connected graphs, this improves the above-mentioned result of Feder and Motwani [5]. Our proof of Theorem 1.1 implies a cubic algorithm for finding a cycle of length at least $\frac{1}{2} n^{\log _{b} 2}+3$. The multiplicative constant $1 / 2$ and the additive constant 3 are for induction purpose. As in [3], we prove the following three statements simultaneously.

TheOREM 1.2. Let $n \geq 5$ and $d \geq 4$ be integers, let $b=\max \{64,4 d+1\}$ and $r=\log _{b} 2$, and let $G$ be a 3-connected graph with $n$ vertices. Then the following statements hold.

(a) Let $x y \in E(G)$ and $z \in V(G)-\{x, y\}$, and let $t$ denote the number of neighbors of $z$ distinct from $x$ and $y$. Assume $\Delta(G) \leq d+1$, and that every vertex of degree $d+1$ (if any) is incident with edge $z x$ or $z y$. Then there is a cycle $C$ through $x y$ in $G-z$ such that $|C| \geq \frac{1}{2}\left(\frac{(d-1) n}{d t}\right)^{r}+2$.

(b) Suppose $\Delta(G) \leq d$. Then for any distinct e, $f \in E(G)$, there is a cycle $C$ through e and $f$ in $G$ such that $|C| \geq \frac{1}{2}\left(\frac{n}{d}\right)^{r}+3$.

(c) Suppose $\Delta(G) \leq d$. Then for any $e \in E(G)$, there is a cycle $C$ through $e$ in $G$ such that $|C| \geq \frac{1}{2} n^{r}+3$.

Note the degree condition in (a): $z x$ and $z y$ need not be edges of $G$, but if $x$ (respectively, $y$ ) has degree $d+1$, then $z x$ (respectively, $z y$ ) must be an edge of $G$, and if $z$ has degree $d+1$, then $z x$ or $z y$ must be an edge of $G$. This condition is due to the addition of edges in order to maintain 3-connectivity.

When $n \geq 5$, Theorem 1.2(c) clearly implies Theorem 1.1. When $n=4$, Theorem 1.1 is obvious. The next result says that Theorem 1.2 holds for graphs with bounded size, which will enable us to avoid dealing with small graphs in inductive proofs. We omit its proof, since it is rather straightforward.

Lemma 1.3. Let $G, n, d, b, r$ be the same as in Theorem 1.2. If $n \leq 4 d+1$, then Theorem 1.2(a) and (b) hold, and if $n \leq(4 d+1)^{2}$, then Theorem 1.2(c) holds.

To prove Theorem 1.2, we need to deal with graphs obtained from 3-connected graphs by deleting a vertex (such as $G-z$ in (a)); such graphs need not be 3 -connected. By using a result of Tutte [11] and an algorithm of Hopcroft and Tarjan [7], we can decompose such graphs into "3-connected components." We then find long paths through certain 3-connected components and use properties of the function $x^{\log _{b} 2}$ to account for the unused 3-connected components. (For a brief outline of our approach, the reader is referred to the algorithm in section 6.) Our approach is similar to that in [3], but here we prove stronger properties of the function $x^{\log _{b} 2}$ and analyze the 3 -connected components in a more sophisticated way.

We organize this paper as follows. In section 2, we recall notation of Hopcroft and Tarjan [7] concerning the decomposition result of Tutte [11] of 2-connected graphs into 3 -connected components. We then define cycle chains of 3-connected components, and prove several results on paths in cycle chains. We prove in section 3 several useful properties of the function $f(x)=x^{\log _{b} 2}$. We also define block chains of 3-connected components, and prove lemmas concerning paths in block chains. Theorem 1.2 will be shown inductively. So in sections 4 and 5 , we show how to reduce Theorem 1.2 to smaller graphs. In section 6 , we complete the proof of our main result, and outline a cubic algorithm for finding a long cycle in a 3-connected graph with bounded degree. 
For graphs $G$ and $H$, we use $G \cong H$ (respectively, $G \neq H$ ) to mean that $G$ is isomorphic to (respectively, not isomorphic to) $H$. Let $G$ be a graph, $H$ a subgraph of $G$, and $S:=\left\{v_{1}, \ldots, v_{k}, x_{1} y_{1}, \ldots, x_{p} y_{p}\right\}$, where $v_{i}, x_{j}, y_{j}$ are vertices of $G$ and $\left\{x_{1}, y_{1}, \ldots, x_{p}, y_{p}\right\} \subseteq\left\{v_{1}, \ldots, v_{k}\right\} \cup V(H)$. Then $H+S$ denotes the simple graph with $V(H+S):=V(H) \cup\left\{v_{1}, \ldots, v_{k}\right\}$ and $E(H+S)=E(H) \cup\left\{x_{1} y_{1}, \ldots, x_{p} y_{p}\right\}$.

2. Paths in cycle chains. For convenience, we recall the decomposition of a 2-connected graph into 3-connected components. A detailed description can be found in [3] and [7].

Let $G$ be a 2-connected graph. We allow multiple edges for the description of this decomposition. Then, $E(G)$ in this section is treated as a multi-set. We say that $\{a, b\} \subseteq V(G)$ is a separation pair in $G$ if there are subgraphs $G_{1}, G_{2}$ of $G$ such that $G_{1} \cup G_{2}=G, V\left(G_{1} \cap G_{2}\right)=\{a, b\}, E\left(G_{1} \cap G_{2}\right)=\emptyset$, and $\left|E\left(G_{i}\right)\right| \geq 2$ for $i=1,2$. Let $G_{i}^{\prime}:=\left(V\left(G_{i}\right), E\left(G_{i}\right) \cup\{a b\}\right)$ for $i=1,2$. Then $G_{1}^{\prime}$ and $G_{2}^{\prime}$ are called split graphs of $G$ with respect to the separation pair $\{a, b\}$, and the new edge $a b$ added to $G_{i}$ is called a virtual edge. It is easy to see that, since $G$ is 2-connected, $G_{i}^{\prime}$ is 2-connected or $G_{i}^{\prime}$ consists of two vertices and at least three multiple edges between them.

Suppose a multigraph is split, and the split graphs are split, and so on, until no more splits are possible. Then each remaining graph is called a split component. No split component contains a separation pair and, therefore, each split component must be one of the following: a triangle, a triple bond (two vertices and three multiple edges between them), or a 3-connected graph.

It is not hard to see that if a split component of a 2-connected graph is 3connected, then it is uniquely determined. It is also easy to see that, for any two split components $G_{1}, G_{2}$ of a 2-connected graph, we have $\left|V\left(G_{1} \cap G_{2}\right)\right| \leq 2$, and if $\left|V\left(G_{1} \cap G_{2}\right)\right|=2$, then either $G_{1}$ and $G_{2}$ share a virtual edge between the vertices in $V\left(G_{1} \cap G_{2}\right)$ or there is a sequence of triple bonds such that the first shares a virtual edge with $G_{1}$, any two consecutive triple bonds in the sequence share a virtual edge, and the last triple bond shares a virtual edge with $G_{2}$.

In order to make such decomposition unique, some triple bonds and triangles need to be merged. Let $G_{i}^{\prime}=\left(V_{i}^{\prime}, E_{i}^{\prime}\right), i=1,2$, be two split components, both containing a virtual edge $a b$. Let $G^{\prime}=\left(V_{1}^{\prime} \cup V_{2}^{\prime},\left(E_{1}^{\prime}-\{a b\}\right) \cup\left(E_{2}^{\prime}-\{a b\}\right)\right)$. The graph $G^{\prime}$ is called the merge graph of $G_{1}^{\prime}$ and $G_{2}^{\prime}$. Clearly, a merge of triple bonds gives a graph consisting of two vertices and multiple edges, which is called a bond. Also a merge of triangles gives a cycle, and a merge of cycles gives a cycle as well.

Let $\mathcal{D}$ denote the set of those 3 -connected split components of a 2-connected graph $G$. We merge the split components of $G$ not in $\mathcal{D}$ as follows: the bonds are merged as much as possible to give a set of bonds $\mathcal{B}$, and the cycles are merged as much as possible to give a set of cycles $\mathcal{C}$. Then $\mathcal{B} \cup \mathcal{C} \cup \mathcal{D}$ is the set of the 3 -connected components of $G$. Note that any two 3-connected components either are edge disjoint or share exactly one virtual edge. The following theorem is a combination of a result of Tutte [11] and an algorithm of Hopcroft and Tarjan [7].

THEOREM 2.1. The 3-connected components of any 2-connected graph are unique and can be found in $O(E)$ time.

If we define a graph whose vertices are the 3-connected components of $G$ and, where two vertices are adjacent whenever the corresponding 3-connected components share a virtual edge, then this graph is a tree, which we call the block-bond tree of $G$. For convenience, 3-connected components that are not bonds are called 3-blocks. An extreme 3 -block is a 3-block that contains at most one virtual edge. That is, either it is the only 3-connected component (in which case $G$ is 3 -connected), or it corresponds 
to a degree one vertex in the block-bond tree.

A cycle chain in a 2-connected graph $G$ is a sequence $C_{1} C_{2} \ldots C_{k}$ of 3-blocks of $G$ such that each $C_{i}$ is a cycle and there exist bonds (possibly empty) $B_{1}, B_{2}, \ldots, B_{k-1}$ in $G$ such that $C_{1} B_{1} C_{2} B_{2} \ldots B_{k-1} C_{k}$ is a path in the block-bond tree of $G$. For convenience, we sometimes write $H:=C_{1} \ldots C_{k}$ for a cycle chain, and view $H$ as the simple graph obtained from the union of $C_{i}(1 \leq i \leq k)$ by identifying virtual edges between the vertices of $C_{i} \cap C_{i+1}(1 \leq i \leq k-1)$. The following is a direct consequence of the definition of a cycle chain.

Proposition 2.2. Let $G$ be a 2-connected graph and $H:=C_{1} \ldots C_{k}$ be a cycle chain in $G$. Then deleting all edges of $H$ with both ends in $V\left(C_{i} \cap C_{i+1}\right), 1 \leq i \leq k-1$, results in a cycle.

The next result finds a path linking two edges in a cycle chain.

Proposition 2.3. Let $G$ be a 2-connected graph, let $H:=C_{1} \ldots C_{k}$ be a cycle chain in $G$, let $u v \in E\left(C_{1}\right)$ with $\{u, v\} \neq V\left(C_{1} \cap C_{2}\right)$ when $k \neq 1$, and let $a b \in E\left(C_{k}\right)$ with $\{a, b\} \neq V\left(C_{k-1} \cap C_{k}\right)$ when $k \neq 1$. Then there is a path in $H-\{v, a b\}$ from $u$ to $\{a, b\}$ and containing $V\left(\bigcup_{i=1}^{k-1}\left(C_{i} \cap C_{i+1}\right)\right)-(\{a, b\} \cup\{u, v\})$.

Proof. We apply induction on $k$. The result holds trivially for $k=1$. So assume $k \geq 2$. Let $H^{\prime}:=C_{2} \ldots C_{k}$ and $V\left(C_{1} \cap C_{2}\right)=\left\{u_{1}, v_{1}\right\}$. Without loss of generality, we may assume that $C_{1}-\left\{v, v_{1}\right\}$ contains a path $P$ from $u$ to $u_{1}$. Suppose $v_{1}=v$. By induction, we find a path $Q$ in $H^{\prime}-\left\{v_{1}, a b\right\}$ from $u_{1}$ to $\{a, b\}$ and containing $V\left(\bigcup_{i=2}^{k-1}\left(C_{i} \cap C_{i+1}\right)\right)-\left(\{a, b\} \cup\left\{u_{1}, v_{1}\right\}\right)$. Then $P \cup Q$ gives the desired path. Now assume $v_{1} \neq v$. By induction, we find a path $Q^{\prime}$ in $H^{\prime}-\left\{u_{1}, a b\right\}$ from $v_{1}$ to $\{a, b\}$ and containing $V\left(\bigcup_{i=2}^{k-1}\left(C_{i} \cap C_{i+1}\right)\right)-\left(\{a, b\} \cup\left\{u_{1}, v_{1}\right\}\right)$. Now $\left(P \cup Q^{\prime}\right)+u_{1} v_{1}$ gives the desired path.

Remark. The path, say $R$, found in Proposition 2.3 may use edges between the vertices of $C_{i} \cap C_{i+1}(1 \leq i \leq k-1)$. However, either $G$ has an edge between the vertices of $C_{i} \cap C_{i+1}$, or $C_{i} \cap C_{i+1}$ is contained in a 3-block of $G$ not in $\mathcal{H}$. Hence, from $R$ we can produce a path in $G$ by replacing virtual edges in $R$ with appropriate paths in $G$, and this new path is at least as long as $R$. This observation applies to the next three results as well, and will be frequently used.

A similar argument establishes the following result, which finds a path in a cycle chain between two vertices and avoiding a specific vertex.

Proposition 2.4. Let $G$ be a 2-connected graph, let $H:=C_{1} \ldots C_{k}$ be a cycle chain in $G$, let $u v \in E\left(C_{1}\right)$ with $\{u, v\} \neq V\left(C_{1} \cap C_{2}\right)$ when $k \neq 1$, and let $x \in V\left(C_{k}\right)$ with $x \neq v$ when $k=1$ and $x \notin V\left(C_{k-1} \cap C_{k}\right)$ when $k \neq 1$. Then there is a path in $H-v$ from $u$ to $x$ and containing $V\left(\bigcup_{i=1}^{k-1}\left(C_{i} \cap C_{i+1}\right)\right)-\{v\}$.

It is clear that the paths and cycle in the above three propositions can be found in $O(V)$ time. The following two results are Propositions 2.7 and 2.8 in [3], which find in $O(V)$ time paths through a given edge in a cycle chain.

Proposition 2.5. Let $G$ be a 2-connected graph, let $H:=C_{1} \ldots C_{k}$ be a cycle chain in $G$, let $u v \in E\left(C_{1}\right)$ with $\{u, v\} \neq V\left(C_{1} \cap C_{2}\right)$ when $k \neq 1$, ab $\in E\left(C_{k}\right)$ with $\{a, b\} \neq V\left(C_{k-1} \cap C_{k}\right)$ when $k \neq 1$, and $c d \in E\left(\bigcup_{i=1}^{k} C_{i}\right)-\{a b\}$. Suppose $a b \neq u v$ when $k=1$. Then there is a path $P$ in $H-a b$ from $\{a, b\}$ to $\{c, d\}$ such that $u v \in E(P), c d \notin E(P)$ unless $c d=u v$, and $V\left(\bigcup_{i=1}^{k-1}\left(C_{i} \cap C_{i+1}\right)\right) \subseteq V(P)$.

Proposition 2.6. Let $G$ be a 2-connected graph, let $H:=C_{1} \ldots C_{k}$ be a cycle chain in $G$, let $u v \in E\left(C_{1}\right)$ with $\{u, v\} \neq V\left(C_{1}\right) \cap V\left(C_{2}\right)$ when $k \neq 1, x \in V\left(C_{k}\right)$ with $x \notin V\left(C_{k-1} \cap C_{k}\right)$ when $k \neq 1$, and $c d \in E\left(\bigcup_{i=1}^{k} C_{i}\right)$. Then there is a path $P$ in $H$ from $x$ to $\{c, d\}$ such that $u v \in E(P), c d \notin E(P)$ unless $c d=u v$, and 
$V\left(\bigcup_{i=1}^{k-1}\left(C_{i} \cap C_{i+1}\right)\right) \subseteq V(P)$

We conclude this section by recalling from [3] two graph operations and three lemmas. Let $G$ be a graph and let $e, f$ be distinct edges of $G$. An H-transform of $G$ at $\{e, f\}$ is an operation that subdivides $e$ and $f$ by vertices $x$ and $y$, respectively, and then adds the edge $x y$. Let $x \in V(G)$ such that $x$ is not incident with $e$. A T-transform of $G$ at $\{x, e\}$ is an operation that subdivides $e$ with a vertex $y$ and then adds the edge $x y$. If there is no need to specify $e, f, x$, we simply speak of an $\mathrm{H}$-transform or a T-transform. The following result is Lemma 3.3 in [3].

Lemma 2.7. Let $d \geq 3$ be an integer and let $G$ be a 3-connected graph with $\Delta(G) \leq d$. Let $G^{\prime}$ be a graph obtained from $G$ by an $H$-transform or a T-transform. Then $G^{\prime}$ is a 3 -connected graph, the vertex of $G$ involved in the T-transform has degree at most $d+1$, and all other vertices of $G^{\prime}$ have degree at most $d$.

The next two results are Lemmas 3.6 and 3.7 in [3], where it is shown that the path $P$ can be found in $O(V)$ time.

Lemma 2.8. Let $G$ be a 3-connected graph, let $f \in E(G)$, let ab, cd, vw $\in E(G)-$ $\{f\}$, and assume that $\{c, d\} \neq\{v, w\}$. Then there exists a path $P$ in $G$ from $\{a, b\}$ to some $z \in\{c, d\} \cup\{v, w\}$ such that (i) $f \in E(P)$, (ii) $c d \in E(P)$ or $v w \in E(P)$, (iii) if $c d \in E(P)$, then $z \in\{v, w\}$ and $v w \notin E(P)$, and (iv) if $v w \in E(P)$, then $z \in\{c, d\}$ and $c d \notin E(P)$.

Lemma 2.9. Let $G$ be a 3-connected graph, let $f \in E(G)$, let $x \in V(G)$ such that $x$ is not incident with $f$, let $c d, v w \in E(G)-\{f\}$, and assume that $\{c, d\} \neq\{v, w\}$. Then there exists a path $P$ in $G$ from $x$ to some $z \in\{c, d\} \cup\{v, w\}$ such that (i) $f \in E(P)$, (ii) $c d \in E(P)$ or $v w \in E(P)$, (iii) if $c d \in E(P)$, then $z \in\{v, w\}$ and $v w \notin E(P)$, and (iv) if $v w \in E(P)$, then $z \in\{c, d\}$ and $c d \notin E(P)$.

3. Paths in block chains. We first prove four lemmas concerning the function $x^{\log _{b} 2}$. These lemmas will then be used to find long paths in block chains. First, we recall Lemma 3.1 in [3].

Lemma 3.1. Let $b \geq 4$ be an integer, and let $m \geq n$ be positive integers. Then $m^{\log _{b} 2}+n^{\log _{b} 2} \geq(m+(b-1) n)^{\log _{b} 2}$.

When $m$ is sufficiently larger than $n$, we have the following result.

LEMma 3.2. Let $b \geq 9$ be an integer, let $m$ and $n$ be positive integers, and assume $m \geq \frac{b(b-1)}{4} n$. Then $m^{\log _{b} 2}+n^{\log _{b} 2} \geq\left(m+\frac{b(b-1)}{4} n\right)^{\log _{b} 2}$.

Proof. By dividing $m^{\log _{b} 2}$ to the above inequality, we see what we need to prove is equivalent to the statement: for any $0 \leq s \leq \frac{4}{b(b-1)}, 1+s^{\log _{b} 2} \geq\left(1+\frac{b(b-1)}{4} s\right)^{\log _{b} 2}$.

Let $f(s)=1+s^{\log _{b} 2}-\left(1+\frac{b(b-1)}{4} s\right)^{\log _{b} 2}$. Clearly, $f(0)=0$. Note that $b(b-1)>$ $4(b-1)$ when $b \geq 5$. Hence $f(1)=2-\left(1+\frac{b(b-1)}{4}\right)^{\log _{b} 2}<2-b^{\log _{b} 2}=0$. Taking derivative about $s$, we have $f^{\prime}(s)=\left(\log _{b} 2\right)\left(s^{\left(\log _{b} 2\right)-1}-\frac{b(b-1)}{4}\left(1+\frac{b(b-1)}{4} s\right)^{\left(\log _{b} 2\right)-1}\right)$. A simple calculation shows that $f^{\prime}(s)=0$ has a unique solution. Therefore, if $f(c)>0$ for some $0<c<1$, then $f(s) \geq 0$ for all $0 \leq s \leq c$.

Note that $0<\frac{4}{b(b-1)}<1$ and $f\left(\frac{4}{b(b-1)}\right)>1+\left(\frac{1}{b^{2}}\right)^{\log _{b} 2}-2^{\log _{b} 2} \geq 1.25-2^{\log _{9} 2}=$ $0.005587 \ldots>0$. Therefore, we have $f(s) \geq 0$ for all $s \in\left[0, \frac{4}{b(b-1)}\right]$.

When $m$ is not sufficiently larger than $n$, we have the following complementary result.

Lemma 3.3. Let $b \geq 64$ be an integer, let $m \geq n$ be positive integers, and assume $m \leq \frac{b(b-1)}{4} n$. Then $m^{\log _{b} 2}+n^{\log _{b} 2} \geq(4 m)^{\log _{b} 2}$.

Proof. The statement of Lemma 3.3 is equivalent to $1+s^{\log _{b} 2} \geq 4^{\log _{b} 2}$ for all $\frac{4}{b(b-1)} \leq s \leq 1$. Therefore, it suffices to show $1+\left(\frac{4}{b(b-1)}\right)^{\log _{b} 2} \geq 4^{\log _{b} 2}$. This is true 
because $1+\left(\frac{4}{b(b-1)}\right)^{\log _{b} 2} \geq 1+\left(\frac{4}{b^{2}}\right)^{\log _{b} 2}=1+\frac{4^{\log _{b} 2}}{4}>4^{\log _{b} 2}$ (since $b \geq 64$ ).

We shall also use the following observations in the proof of Theorem 1.2.

Lemma 3.4. Let $m$ be an integer, $d \geq 3$, and $b \geq d+1$. If $m \geq 4$, then $m \geq$ $\frac{1}{2} m^{\log _{b} 2}+3$. If $m \geq 3$, then $m>\frac{1}{2}\left(\frac{m}{d}\right)^{\log _{b} 2}+2$. If $m \geq 2$, then $m>\frac{1}{2}\left(\frac{m}{d}\right)^{\log _{b} 2}+1$.

Proof. Let $f(x)=x-\frac{1}{2} x^{\log _{b} 2}$. We can show that $f^{\prime}(x)>0$ for $x \geq 1$. Hence $f(x)$ is an increasing function when $x \geq 1$. Thus, when $x \geq 4$, we have $f(x) \geq f(4)=$ $4-\frac{1}{2} 4^{\log _{b} 2} \geq 3$ (since $b \geq 4$ ). The first inequality holds.

Let $f(x)=x-\frac{1}{2}\left(\frac{x}{d}\right)^{\log _{b} 2}$; then $f(x)$ is increasing when $x \geq 1$. The second inequality follows from $f(3)>2$, and the third inequality follows from $f(2)>1$.

We now turn to paths in block chains. Let $G$ be a 2-connected graph. A block chain in $G$ is a sequence $H_{1} \ldots H_{h}$ for which (1) each $H_{i}$ is either a cycle chain in $G$ or a 3-connected 3-block of $G,(2)$ for any $1 \leq s \leq h-1, H_{s}$ or $H_{s+1}$ is 3-connected, and (3) there exist bonds (possibly empty) $B_{1}, \ldots, B_{h-1}$ such that $H_{1} B_{1} H_{2} B_{2} \ldots B_{h-1} H_{h}$ form a path in the block-bond tree of $G$ (by also including the tree paths corresponding to $H_{i}$ when $H_{i}$ is a cycle chain). A detailed description with examples can be found in [3]. For convenience, we sometimes write $\mathcal{H}:=H_{1} \ldots H_{h}$ for a block chain and view $\mathcal{H}$ as the simple graph obtained from $\bigcup_{i=1}^{n} H_{i}$ by identifying edges between the vertices in $H_{i} \cap H_{i+1}(1 \leq i \leq n-1)$. The edges of $\mathcal{H}$ between the vertices of $H_{i} \cap H_{i+1}$ are called separating edges of $\mathcal{H}$. Such edges are to be avoided when we find paths in block chains.

Let $H_{1} \ldots H_{h}$ be a block chain and let $V\left(H_{s} \cap H_{s+1}\right)=\left\{x_{s}, y_{s}\right\}, 1 \leq s \leq h-1$. For each $1 \leq s \leq h$, we define $A\left(H_{s}\right)$ as follows. If $H_{s}$ is 3-connected, then $A\left(H_{s}\right):=$ $V\left(H_{s}\right)$. If $H_{s}=C_{1} \ldots C_{k}$ is a cycle chain, then let

- $A\left(H_{s}\right):=V\left(\bigcup_{i=1}^{k-1}\left(C_{i} \cap C_{i+1}\right)\right)-\left(\left\{x_{s-1}, y_{s-1}\right\} \cup\left\{x_{s}, y_{s}\right\}\right)$ when $1<s<h$,

- $A\left(H_{s}\right):=V\left(\bigcup_{i=1}^{k-1} C_{i} \cap C_{i+1}\right)$ when $s=1=h, A\left(H_{s}\right):=V\left(\bigcup_{i=1}^{k-1}\left(C_{i} \cap\right.\right.$ $\left.\left.C_{i+1}\right)\right)-\left\{x_{s}, y_{s}\right\}$ when $s=1<h$, and

- $A\left(H_{s}\right):=V\left(\bigcup_{i=1}^{k-1}\left(C_{i} \cap C_{i+1}\right)\right)-\left\{x_{s-1}, y_{s-1}\right\}$ when $1<s=h$.

We write $\sigma(\mathcal{H}):=\sum_{s=1}^{h}\left|A\left(H_{s}\right)\right|$ and $|\mathcal{H}|:=\left|V\left(\bigcup_{i=1}^{h} H_{i}\right)\right|$. For convenience, we define $B_{1}(\mathcal{H})=\left\{H_{i}: H_{i}\right.$ is 3 -connected or $\left.\left|A\left(H_{i}\right)\right| \leq 1\right\}$ and $B_{2}(\mathcal{H})=\left\{H_{i}: H_{i}\right.$ is a cycle chain and $\left.\left|A\left(H_{i}\right)\right| \geq 2\right\}$.

In the remainder of this section, we show how to find long paths in block chains (in terms of $\sigma(\mathcal{H})$ ). All proofs imply $O(V)$ algorithms that reduce the problem of finding a path to Theorem 1.2 for smaller graphs.

Lemma 3.5. Let $n \geq 6$ be an integer and assume Theorem 1.2 holds for graphs with at most $n-1$ vertices. Let $\mathcal{H}:=H_{1} H_{2} \ldots H_{h}$ be a block chain in a 2-connected graph such that $|\mathcal{H}|<n$ and $\Delta\left(H_{i}\right) \leq d$ for $1 \leq i \leq h$. Let $u v \in E\left(H_{1}\right)$ such that $\{u, v\}$ is not a cut of $H_{1}$, and if $h \geq 2$, then $\{u, v\} \neq V\left(H_{1} \cap H_{2}\right)$. Then there is a path $P$ in $\mathcal{H}$ from $u$ to $v$ such that $|E(P)| \geq \frac{1}{2}\left(\frac{(d-1) \sigma(\mathcal{H})}{d}\right)^{r}+2$ and $P$ contains no separating edge of $\mathcal{H}$.

Proof. When $h \geq 2$, we use $a, b$ to denote the vertices in $V\left(H_{1} \cap H_{2}\right)$. Suppose $\left|A\left(H_{1}\right)\right| \geq \frac{(d-1) \sigma(\mathcal{H})}{d}$. First assume $H_{1}$ is a cycle chain or $H_{1} \cong K_{4}$. Then there is a Hamilton path $P_{1}$ in $H_{1}$ from $u$ to $v$ (by Proposition 2.2 when $H_{1}$ is a cycle chain). If $\left|H_{1}\right|=3$, then $\left|A\left(H_{1}\right)\right|=0$, and hence, $\left|E\left(P_{1}\right)\right| \geq \frac{1}{2}\left|A\left(H_{1}\right)\right|^{r}+2$. If $\left|H_{1}\right| \geq 4$, then $\left|E\left(P_{1}\right)\right| \geq 3$, and by Lemma 3.4, $\left|E\left(P_{1}\right)\right| \geq \frac{1}{2}\left|H_{1}\right|^{r}+2 \geq \frac{1}{2}\left|A\left(H_{1}\right)\right|^{r}+2$. Now assume $H_{1}$ is 3 -connected and $H_{1} \neq K_{4}$. Then by Theorem $1.2(\mathrm{c}), H_{1}$ has a cycle $C_{1}$ through $u v$ such that $\left|E\left(C_{1}\right)\right| \geq \frac{1}{2}\left|H_{1}\right|^{r}+3=\frac{1}{2}\left|A\left(H_{1}\right)\right|^{r}+3$. Let $P_{1}:=C_{1}-u v$. If $h=1$ or $a b \notin E\left(P_{1}\right)$, then $P:=P_{1}$ gives the desired path. If $h \geq 2$ and $a b \in E\left(P_{1}\right)$, then by replacing $a b$ with a path in $H_{2} \ldots H_{h}$ between $a$ and $b$ and not containing 
any separating edge of $\mathcal{H}$, we obtain the desired path $P$.

So we may assume $\left|A\left(H_{1}\right)\right|<\frac{(d-1) \sigma(\mathcal{H})}{d}$. In particular, $h \geq 2$. If $H_{1}$ is a cycle chain or $H_{1} \cong K_{4}$, then, as in the above paragraph, we find a Hamilton path $P_{1}$ from $u$ to $v$ in $H_{1}$ through $a b$ such that $\left|E\left(P_{1}\right)\right| \geq \frac{1}{2}\left|A\left(H_{1}\right)\right|^{r}+2$. Now assume $H_{1}$ is 3-connected and $H_{1} \neq K_{4}$. Then by Theorem 1.2(b), $H_{1}$ has a cycle $C_{1}$ through $u v$ and $a b$ such that $\left|E\left(C_{1}\right)\right| \geq \frac{1}{2}\left(\frac{\left|A\left(H_{1}\right)\right|}{d}\right)^{r}+3$; let $P_{1}:=C_{1}-u v$.

By induction, we find a path $P^{\prime}$ in $\mathcal{H}^{\prime}:=H_{2} \ldots H_{h}$ from $a$ to $b$ and containing no separating edges of $\mathcal{H}^{\prime}$ such that $\left|E\left(P^{\prime}\right)\right| \geq \frac{1}{2}\left(\frac{(d-1) \sigma\left(\mathcal{H}^{\prime}\right)}{d}\right)^{r}+2$. Let $P:=\left(P_{1}-a b\right) \cup P^{\prime}$. Since $\sigma(\mathcal{H}) \leq A\left(H_{1}\right)+\sigma\left(\mathcal{H}^{\prime}\right)$ and $\left|A\left(H_{1}\right)\right|<\frac{(d-1) \sigma(\mathcal{H})}{d}, \frac{\left|A\left(H_{1}\right)\right|}{d}<\frac{(d-1) \sigma\left(\mathcal{H}^{\prime}\right)}{d}$. Hence by Lemma 3.2 ,

$$
\begin{aligned}
|E(P)| & >\frac{1}{2}\left(\frac{\left|A\left(H_{1}\right)\right|}{d}\right)^{r}+\frac{1}{2}\left(\frac{(d-1) \sigma\left(\mathcal{H}^{\prime}\right)}{d}\right)^{r}+2 \\
& \geq \frac{1}{2}\left((b-1) \frac{\left|A\left(H_{1}\right)\right|}{d}+\frac{(d-1) \sigma\left(\mathcal{H}^{\prime}\right)}{d}\right)^{r}+2 \\
& >\frac{1}{2}\left(\frac{(d-1) \sigma(\mathcal{H})}{d}\right)^{r}+2 .
\end{aligned}
$$

So $P$ gives the desired path.

For the next two lemmas, we define $u v$ and $x$ in a block chain $\mathcal{H}:=H_{0} H_{1} \ldots H_{h}$ (in a 2-connected graph). Suppose $h=0$. If $H_{0}$ is 3 -connected or $H_{0}$ is a cycle, then let $u v \in E\left(H_{0}\right)$ and $x \in V\left(H_{0}\right)-\{u, v\}$, and if $H_{0}=C_{1} \ldots C_{k}$ is a cycle chain with $k \geq 2$, then let $u v \in E\left(C_{1}\right)$ with $\{u, v\} \neq V\left(C_{1} \cap C_{2}\right)$ and let $x \in V\left(C_{k}\right)-V\left(C_{k-1}\right)$. Now assume $h \geq 1$. If $H_{0}$ is 3 -connected or $H_{0}$ is a cycle, then let $u v \in E\left(H_{0}\right)$ with $\{u, v\} \neq V\left(H_{0} \cap H_{1}\right)$; if $H_{0}=C_{1} \ldots C_{k}$ is a cycle chain with $k \geq 2$ and $V\left(H_{0} \cap H_{1}\right)=$ $V\left(C_{k} \cap H_{1}\right)$, then let $u v \in E\left(C_{1}\right)$ with $\{u, v\} \neq V\left(C_{1} \cap C_{2}\right)$; if $H_{h}$ is a cycle or $H_{h}$ is 3-connected, then let $x \in V\left(H_{h}\right)-V\left(H_{h-1}\right)$; and if $H_{h}=C_{1} \ldots C_{k}$ is a cycle chain with $k \geq 2$ and $V\left(H_{h-1} \cap H_{h}\right)=V\left(H_{h-1} \cap C_{1}\right)$, then let $x \in V\left(C_{k}\right)-V\left(C_{k-1}\right)$.

LEMMA 3.6. Let $n \geq 6$ be an integer and assume Theorem 1.2 holds for graphs with at most $n-1$ vertices. Let $\mathcal{H}:=H_{0} H_{1} \ldots H_{h}$, uv, $x$ be defined as above, and assume $|\mathcal{H}|<n, \Delta\left(H_{i}\right) \leq d$ for $0 \leq i \leq h$, and the degree of $x$ in $H_{h}$ is at most $d-1$. Then there exists a path $P$ in $\mathcal{H}-v$ from $u$ to $x$ and containing no separating edge of $\mathcal{H}$ such that

(i) $|E(P)| \geq \frac{1}{2}\left(\sum_{i=0}^{h}\left(\frac{\left|A\left(H_{i}\right)\right|}{d}\right)^{r}\right)+1 \geq \frac{1}{2}\left(\frac{\sigma(\mathcal{H})}{d}\right)^{r}+1$, and

(ii) $|E(P)| \geq \frac{1}{2}\left(\sum\left\{\left(\frac{\left|A\left(H_{i}\right)\right|}{d}\right)^{r}: H_{i} \in B_{1}(\mathcal{H})\right\}\right)+\left(\sum\left\{\max \left\{1,\left|A\left(H_{i}\right)\right|-2\right\}: H_{i} \in\right.\right.$ $\left.\left.B_{2}(\mathcal{H})\right\}\right)+1$.

Proof. We apply induction on $h$. Suppose $h=0$. If $H_{0}$ is 3-connected and $H_{0} \approx K_{4}$, then by assumption and because $x$ has degree at most $d-1$, Theorem $1.2(\mathrm{a})$ holds for $H_{0}+\{v x, u x\}$. Hence, $H_{0}-v$ contains a path $P$ from $u$ to $x$ such that $|E(P)| \geq \frac{1}{2}\left(\frac{\left|A\left(H_{0}\right)\right|}{d}\right)^{r}+1$. If $H_{0} \cong K_{4}$, then we can find a path $P$ from $u$ to $x$ in $H_{0}-v$ such that $|E(P)|=2 \geq \frac{1}{2}\left(\frac{\left|A\left(H_{0}\right)\right|}{d}\right)^{r}+1$. If $H_{0}$ is a cycle chain, then by Proposition 2.4, there is a path $P$ from $u$ to $x$ in $H_{0}-v$ containing $A\left(H_{0}\right)-\{v\}$. Note that $x \notin A\left(H_{0}\right)$ and if $v \in A\left(H_{0}\right)$, then $u \notin A\left(H_{0}\right)$. Thus, $|E(P)| \geq\left|A\left(H_{0}\right)\right|$. Because $|E(P)| \geq 1$ and since $\left|A\left(H_{0}\right)\right|=0$ or $\left|A\left(H_{0}\right)\right| \geq 2$, we have $|E(P)| \geq \frac{1}{2}\left(\frac{\left|A\left(H_{0}\right)\right|}{d}\right)^{r}+1$ (by Lemma 3.4). Clearly, $|E(P)| \geq \max \left\{1,\left|A\left(H_{0}\right)\right|-2\right\}+1$ when $H_{0} \in B_{2}(\mathcal{H})$.

Now assume $h \geq 1$. Let $V\left(H_{0} \cap H_{1}\right)=\left\{u_{0}, v_{0}\right\}$, and assume the notation is chosen so that $u_{0} \notin\{u, v\}$. By the above argument for $h=0$, if $H_{0}$ is a cycle chain or $H_{0} \cong K_{4}$, then $H_{0}-v$ has a path $P_{0}$ from $u$ to $u_{0}$ such that $\left|E\left(P_{0}\right)\right| \geq \frac{1}{2}\left(\frac{\left|A\left(H_{0}\right)\right|}{d}\right)^{r}+1$, 
and $\left|E\left(P_{0}\right)\right| \geq \max \left\{1,\left|A\left(H_{0}\right)\right|-2\right\}+1$ when $H_{0} \in B_{2}(\mathcal{H})$. (Note in the case $H_{0}$ is a cycle chain, $u_{0} \notin A\left(H_{0}\right)$ because $h \geq 1$.) Now assume $H_{0}$ is 3 -connected and $\left|H_{0}\right| \geq 5$. If $v=v_{0}$, then we apply Theorem 1.2(a) to find a path $P_{0}$ from $u$ to $u_{0}$ in $\left(H_{0}+u u_{0}\right)-v$ such that $\left|E\left(P_{0}\right)\right| \geq \frac{1}{2}\left(\frac{\left|A\left(H_{0}\right)\right|}{d}\right)^{r}+1$. If $v \neq v_{0}$, then let $H_{0}^{\prime}$ be obtained from $H_{0}$ by a T-transform at $\left\{v, u_{0} v_{0}\right\}$ and let $u^{\prime}$ denote the new vertex. By Theorem 1.2(a), we find a path $P_{0}^{*}$ in $\left(H_{0}^{\prime}+u u^{\prime}\right)-v$ from $u$ to $u^{\prime}$ such that $\left|E\left(P_{0}^{*}\right)\right| \geq \frac{1}{2}\left(\frac{\left|A\left(H_{0}\right)\right|}{d}\right)^{r}+1$; and let $P_{0}:=P_{0}^{*}-u^{\prime}$ (in this case $\left.u_{0} v_{0} \notin E\left(P_{0}\right)\right)$.

Let $P_{0}^{\prime}:=P_{0}$ if $u_{0} v_{0} \notin E\left(P_{0}\right)$; otherwise, let $P_{0}^{\prime}:=P_{0}-u_{0}$. Then $P_{0}^{\prime}$ is a path in $H_{0}-\left\{v, u_{0} v_{0}\right\}$ from $u$ to $\left\{u_{0}, v_{0}\right\}$ such that $\left|E\left(P_{0}^{\prime}\right)\right| \geq \frac{1}{2}\left(\frac{\left|A\left(H_{0}\right)\right|}{d}\right)^{r}$, and $\left|E\left(P_{0}^{\prime}\right)\right| \geq$ $\max \left\{1,\left|A\left(H_{0}\right)\right|-2\right\}$ when $H_{0} \in B_{2}(\mathcal{H})$. Without loss of generality, we may assume that $P_{0}^{\prime}$ is from $u_{0}$ to $u$.

By applying induction to $\mathcal{H}^{\prime}:=H_{1} \ldots H_{h}$, there is a path $P_{1}$ from $u_{0}$ to $x$ in $\mathcal{H}^{\prime}-v_{0}$ containing no separating edge of $\mathcal{H}^{\prime}$ such that $\left|E\left(P_{1}\right)\right| \geq \frac{1}{2}\left(\sum_{i=1}^{h}\left(\frac{\left|A\left(H_{i}\right)\right|}{d}\right)^{r}\right)+$ $1 \geq \frac{1}{2}\left(\frac{\sigma\left(\mathcal{H}^{\prime}\right)}{d}\right)^{r}+1$ and $\left|E\left(P_{1}\right)\right| \geq \frac{1}{2}\left(\sum\left\{\left(\frac{\left|A\left(H_{i}\right)\right|}{d}\right)^{r}: H_{i} \in B_{1}(\mathcal{H})\right.\right.$ and $\left.\left.i \neq 0\right\}\right)+$ $\left(\sum\left\{\max \left\{1,\left|A\left(H_{i}\right)\right|-2\right\}: H_{i} \in B_{2}(\mathcal{H})\right.\right.$ and $\left.\left.i \neq 0\right\}\right)+1$.

Let $P:=P_{0}^{\prime} \cup P_{1}$. Because $h \geq 1, H_{0}$ or $H_{1}$ is not a cycle chain, and hence, $\sigma(\mathcal{H}) \leq\left|A\left(H_{0}\right)\right|+\sigma\left(\mathcal{H}^{\prime}\right)$. It is easy to see that $P$ satisfies (i) and (ii). Note that the second inequality in (i) follows from the first in (i) by applying Lemma 3.1.

Lemma 3.7. Assume the same hypothesis of Lemma 3.6. Then for any $0 \leq t \leq h$ and for any $p q \in E\left(H_{t}\right)$ such that $\left|H_{t}\right| \leq n-3$ when $h \geq 1$, there exists a path $P$ in $\mathcal{H}$ from $x$ to $\{p, q\}$ and containing no separating edge of $\mathcal{H}$ such that

(i) $p q \notin E(P)$, and $|E(P)| \geq \frac{1}{2}\left|A\left(H_{0}\right)\right|^{r}+\frac{1}{2}\left(\sum\left\{\left(\frac{\left|A\left(H_{i}\right)\right|}{d}\right)^{r}: H_{i} \in B_{1}(\mathcal{H})\right.\right.$ and $i \neq 0\})+\left(\sum\left\{\max \left\{1,\left|A\left(H_{i}\right)\right|-2\right\}: H_{i} \in B_{2}(\mathcal{H})\right.\right.$ and $\left.\left.i \neq 0\right\}\right)+1$, and

(ii) if we require uv $\in E(P)$, then $p q \notin E(P)$ unless $p q=u v$, and $|E(P)| \geq$ $\frac{1}{2}\left(\sum\left\{\left(\frac{\left|A\left(H_{i}\right)\right|}{d}\right)^{r}: H_{i} \in B_{1}(\mathcal{H})\right\}\right)+\left(\sum\left\{\max \left\{1,\left|A\left(H_{i}\right)\right|-2\right\}: H_{i} \in B_{2}(\mathcal{H})\right\}\right)+$ $1 \geq \frac{1}{2}\left(\frac{\sigma(\mathcal{H})}{d}\right)^{r}+1$.

Proof. We apply induction on $h$. Note that the second inequality in (ii) follows from the first in (ii) by applying Lemma 3.1.

Case $1 . h=0$.

First, assume $H_{0}$ is a cycle chain. Then by Proposition 2.6, there is a path $P$ from $x$ to $\{p, q\}$ in $H_{0}$ such that $u v \in E(P), p q \notin E(P)$ unless $p q=u v$, and $A\left(H_{0}\right) \subseteq V(P)$. Because $x \notin A\left(H_{0}\right),|E(P)| \geq\left|A\left(H_{0}\right)\right|$. Because $x \notin\{u, v\},|E(P)| \geq 2$. So $|E(P)| \geq$ $\max \left\{1,\left|A\left(H_{0}\right)\right|-2\right\}+1$. Moreover, if $\left|A\left(H_{0}\right)\right| \leq 3$, then $|E(P)| \geq 2>\frac{1}{2}\left|A\left(H_{0}\right)\right|^{r}+$ 1 , and if $\left|A\left(H_{0}\right)\right| \geq 4$, then by Lemma 3.4 we have $|E(P)| \geq\left|A\left(H_{0}\right)\right| \geq \frac{1}{2}\left|A\left(H_{0}\right)\right|^{r}+3$. Clearly (i) and (ii) hold.

Now assume $H_{0} \cong K_{4}$. Let $P$ denote a Hamilton path in $H_{0}$ from $x$ to $\{p, q\}$ such that $u v \in E(P)$, and $p q \notin E(P)$ unless $p q=u v$. Then $|E(P)|=3>\frac{1}{2}\left|A\left(H_{0}\right)\right|^{r}+1$ and (i) and (ii) hold.

Finally, assume $H_{0}$ is 3-connected and $H_{0} \nsucceq K_{4}$. Then $5 \leq\left|H_{0}\right|<n$. If $x \in\{p, q\}$, then we apply Theorem 1.2(c) (respectively, Theorem 1.2(b)) to find a cycle $C$ through $p q$ (respectively, $p q$ and $u v$ ) such that $|C| \geq \frac{1}{2}\left|A\left(H_{0}\right)\right|^{r}+3$ (respectively, $\left.|C| \geq \frac{1}{2}\left(\frac{\left|A\left(H_{0}\right)\right|}{d}\right)^{r}+3\right)$. Now it is easy to see that (i) and (ii) hold with $P:=C-p q$. So assume $x \notin\{p, q\}$. Then let $H_{0}^{\prime}$ be obtained from $H_{0}$ by a T-transform at $\{x, p q\}$ and let $x^{\prime}$ denote the new vertex. By Theorem 1.2(c) (respectively, Theorem 1.2(b)), we find a cycle $C$ through $x x^{\prime}$ (respectively, $x x^{\prime}$ and $u v$ ) such that $|C| \geq \frac{1}{2}\left|H_{0}\right|^{r}+3$ (respectively, $|C| \geq \frac{1}{2}\left(\frac{\left|H_{0}\right|}{d}\right)^{r}+3$ ). Now it is easy to see that (i) and (ii) hold with $P:=C-x^{\prime}$. 
Case $2 . h \geq 1$.

Let $\{a, b\}=V\left(H_{0} \cap H_{1}\right)$.

Suppose $p q \in \mathcal{H}^{\prime}:=H_{1} \ldots H_{h}$. By applying induction to $\mathcal{H}^{\prime}$ (with $a b$ playing the role of $u v$ ), we find a path $P^{\prime}$ in $\mathcal{H}^{\prime}$ from $x$ to $\{p, q\}$ and containing no separating edge of $\mathcal{H}^{\prime}$ such that $a b \in E\left(P^{\prime}\right), p q \notin E\left(P^{\prime}\right)$ unless $p q=a b$, and $\left|E\left(P^{\prime}\right)\right| \geq$ $\frac{1}{2}\left(\sum\left\{\left(\frac{\left|A\left(H_{i}\right)\right|}{d}\right)^{r}: H_{i} \in B_{1}(\mathcal{H})\right.\right.$ and $\left.\left.i \neq 0\right\}\right)+\left(\sum\left\{\max \left\{1,\left|A\left(H_{i}\right)\right|-2\right\}: H_{i} \in B_{2}(\mathcal{H})\right.\right.$ and $i \neq 0\})+1$. If $H_{0}$ is a cycle chain or $H_{0} \cong K_{4}$, then $H_{0}$ has a Hamilton cycle $C$ through $a b$ and $u v$. If $H_{0}$ is 3 -connected and $\left|H_{0}\right| \geq 5$, we apply Theorem 1.2(c) (respectively, Theorem 1.2(b)) to find a cycle $C$ through $a b$ (respectively, $a b$ and $u v$ ) such that $|C| \geq \frac{1}{2}\left|H_{0}\right|^{r}+3$ (respectively, $\left.|C| \geq \frac{1}{2}\left(\frac{\left|H_{0}\right|}{d}\right)^{r}+3\right)$. Then $P:=(C-a b) \cup\left(P^{\prime}-a b\right)$ gives the desired path for (i) and (ii).

Therefore, we may assume $p q \in H_{0}$ and $p q \neq a b$. Let $H_{0}^{\prime}$ be obtained from $H_{0}$ by an $H$-transform at $\{p q, a b\}$, and let $a^{\prime}, p^{\prime}$ denote the new vertices. By Theorem 1.2(c) (respectively, Theorem 1.2(b)) we find a cycle $C$ in $H_{0}^{\prime}$ through $a^{\prime} p^{\prime}$ (respectively, $a^{\prime} p^{\prime}$ and $u v$ ) such that $|C| \geq \frac{1}{2}\left|H_{0}\right|^{r}+3$ (respectively, $|C| \geq \frac{1}{2}\left(\frac{\left|H_{0}\right|}{d}\right)^{r}+3$ ). Let $P_{0}:=C-\left\{a^{\prime}, p^{\prime}\right\}$ and, without loss of generality, let $a$ be the end of $P_{0}$. By Lemma 3.6, we can find a path $P^{\prime}$ in $\mathcal{H}^{\prime}-b$ from $x$ to $a$ and containing no separating edge of $\mathcal{H}^{\prime}$ such that $\left|E\left(P^{\prime}\right)\right| \geq \frac{1}{2}\left(\sum\left\{\left(\frac{\left|A\left(H_{i}\right)\right|}{d}\right)^{r}: H_{i} \in B_{1}(\mathcal{H})\right.\right.$ and $\left.\left.i \neq 0\right\}\right)+\left(\sum\left\{\max \left\{1,\left|A\left(H_{i}\right)\right|-2\right\}\right.\right.$ : $H_{i} \in B_{2}(\mathcal{H})$ and $\left.\left.i \neq 0\right\}\right)+1$. Now $P:=P_{0} \cup P^{\prime}$ gives the desired path, except for (ii) when $p q=u v$. In the exceptional case, we may assume $v \notin\{a, b\}$. Let $H_{0}^{\prime \prime}$ be obtained from $H_{0}$ by a T-transform at $\{v, a b\}$, with new vertex $a^{\prime \prime}$. We apply Theorem 1.2(a) to find a cycle $C$ in $\left(H_{0}^{\prime \prime}+u a^{\prime \prime}\right)-v$ through $u a^{\prime \prime}$ such that $|C| \geq \frac{1}{2}\left(\frac{\left|H_{0}\right|}{d}\right)^{r}+2$. Without loss of generality, we may assume $a$ is the end of $C-a^{\prime \prime}$. Let $P^{\prime}$ be found as above. Then $P:=\left(\left(C-a^{\prime \prime}\right) \cup P^{\prime}\right)+\{v, u v\}$ gives the desired path for (ii).

4. Cycles through two edges. We reduce Theorem 1.2(a) and (b) to Theorem 1.2 for smaller graphs. Note that finding a long cycle in Theorem 1.2(a) through $x y$ avoiding $z$ is equivalent to finding a long cycle through edges $x z$ and $y z$. First, we reduce Theorem 1.2(a); our proof implies an $O(E)$ time reduction.

Lemma 4.1. Let $n \geq 6$ be an integer, and assume that Theorem 1.2 holds for graphs with at most $n-1$ vertices. Let $G$ be a 3 -connected graph with $n$ vertices, let $x y \in E(G)$ and $z \in V(G)-\{x, y\}$, and let $t$ denote the number of neighbors of $z$ distinct from $x$ and $y$. Assume $\Delta(G) \leq d+1$, and every vertex of degree $d+1$ in $G$ (if any) is incident with the edge $z x$ or $z y$. Then there is a cycle $C$ through $x y$ in $G-z$ such that $|C| \geq \frac{1}{2}\left(\frac{(d-1) n}{d t}\right)^{r}+2$.

Proof. By Lemma 1.3, we may assume $n \geq 4 d+2$. Since $G$ is 3-connected, $t \geq 1$.

Assume that $G-z$ is 3 -connected. By assumption, $\Delta(G-z) \leq d$. Since $n \geq 6$, $|G-z| \geq 5$. So by Theorem 1.2(c), $G-z$ contains a cycle $C$ through $x y$ such that $|C| \geq \frac{1}{2}(n-1)^{r}+3$. By Lemma $3.1,|C| \geq \frac{1}{2} n^{r}+2>\frac{1}{2}\left(\frac{(d-1) n}{d t}\right)^{r}+2$.

Therefore, we may assume that $G-z$ is not 3 -connected. By Theorem 2.1, we decompose $G-z$ into 3-connected components. Let $\mathcal{H}:=H_{1} \ldots H_{h}$ be a block chain in $G-z$ such that (i) $H_{h}$ contains an extreme 3-block of $G-z$, (ii) $x y \in E\left(H_{1}\right)$ and $\{x, y\} \neq V\left(H_{1}\right) \cap V\left(H_{2}\right)$ when $h \neq 1$, and if $H_{1}=C_{1} \ldots C_{k}$ is a cycle chain with $k \geq 2$ and $V\left(H_{1} \cap H_{2}\right)=V\left(C_{k} \cap H_{2}\right)$ (when $h \neq 1$ ), then $x y \in E\left(C_{1}\right)$ and $\{x, y\} \neq V\left(C_{1} \cap C_{2}\right)$, and (iii) subject to (i) and (ii), $\sigma(\mathcal{H})$ is maximum.

We claim that $\sigma(\mathcal{H}) \geq \frac{n-1-2 t}{t}$. Since $G$ is 3 -connected, each extreme 3-block of $G-z$ distinct from $H_{1}$ contains a neighbor of $z$. Therefore, there are at most $2 t$ degree 2 vertices in $G-z$ and at most $t$ extreme 3-blocks of $G-z$ different from $H_{1}$. Note that the vertices of $G-z$ with degree at least 3 are counted in $\sigma(\mathcal{K})$ for some 
block chain $\mathcal{K}$ (defined as $\mathcal{H}$ above except condition (iii)). It then follows from (iii) that $\sigma(\mathcal{H}) \geq \frac{n-1-2 t}{t}$.

Since $n>4 d+1$ and $t \leq d, \sigma(\mathcal{H}) \geq 2$. By Lemma 3.5, there is a path $P$ from $x$ to $y$ in $\mathcal{H}$ such that $|E(P)| \geq \frac{1}{2}\left(\frac{(d-1) \sigma(\mathcal{H})}{d}\right)^{r}+2$. Let $C^{*}:=P+x y$. Then

$$
\begin{aligned}
\left|C^{*}\right| & =|E(P)|+1 \\
& \geq \frac{1}{2}\left(\frac{(d-1) \sigma(\mathcal{H})}{d}+(b-1)\right)^{r}+2 \quad(\text { by Lemma 3.1) } \\
& \geq \frac{1}{2}\left(\frac{(d-1)(n-1-2 t)+d t(b-1)}{d t}\right)^{r}+2 \\
& >\frac{1}{2}\left(\frac{(d-1) n}{d t}\right)^{r}+2 \quad(\text { since } b \geq 4 d+1) .
\end{aligned}
$$

The desired cycle $C$ can now be obtained from $C^{*}$ by replacing virtual edges in $C^{*}$ with appropriate paths in $G$.

We now reduce Theorem 1.2(b); our proof implies an $O(E)$ time reduction.

Lemma 4.2. Let $n \geq 6$ be an integer, and assume that Theorem 1.2 holds for graphs with at most $n-1$ vertices. Suppose $G$ is a 3 -connected graph on $n$ vertices and $\Delta(G) \leq d$. Then for any $\{e, f\} \subseteq E(G)$, there is a cycle $C$ through $e, f$ in $G$ such that $|C| \geq \frac{1}{2}\left(\frac{n}{d}\right)^{r}+3$.

Proof. By Lemma 1.3, we may assume $n \geq 4 d+2$. First, assume that $e$ is incident with $f$. Let $e=x z$ and $f=y z$, and let $G^{\prime}:=G+x y$. Then $G^{\prime}$ is 3connected, $\Delta\left(G^{\prime}\right) \leq d+1$, and the possible vertices of degree $d+1$ in $G^{\prime}$ are $x$ and $y$. By applying Lemma 4.1 to $G^{\prime}, x y, z$, there is a cycle $C^{\prime}$ through $x y$ in $G^{\prime}-z$ such that $\left|C^{\prime}\right| \geq \frac{1}{2}\left(\frac{(d-1) n}{d t}\right)^{r}+2$, where $t$ is the number of neighbors of $z$ in $G^{\prime}$ distinct from $x$ and $y$. Since $z x, z y \in E(G), t \leq d-1$. Let $C:=\left(C^{\prime}-x y\right)+\{e, f\}$; then $|C| \geq \frac{1}{2}\left(\frac{(d-1) n}{d t}\right)^{r}+3 \geq \frac{1}{2}\left(\frac{n}{d}\right)^{r}+3$. So $C$ gives the desired cycle in $G$.

Therefore, we may assume that $e$ and $f$ are not incident. Let $e=x y$; then $f \in E(G-y)$. Since $G$ is 3 -connected, $G-y$ is 2-connected.

Suppose $G-y$ is 3-connected. Let $y^{\prime} \neq x$ be a neighbor of $y$. Then $G^{\prime}:=$ $(G-y)+x y^{\prime}$ is a 3 -connected graph, $\Delta\left(G^{\prime}\right) \leq d$, and $5 \leq\left|G^{\prime}\right|<n$. By Theorem 1.2(b), there is a cycle $C^{\prime}$ through $x y^{\prime}$ and $f$ in $G^{\prime}$ such that $\left|C^{\prime}\right| \geq \frac{1}{2}\left(\frac{n-1}{d}\right)^{r}+3$. Let $C:=\left(C^{\prime}-x y^{\prime}\right)+\left\{y, x y, y y^{\prime}\right\}$. Then $|C|=\left|C^{\prime}\right|+1 \geq \frac{1}{2}\left(\frac{n-1}{d}\right)^{r}+4$. By Lemma 3.1, $|C| \geq \frac{1}{2}\left(\frac{n}{d}\right)^{r}+3$. So $C$ gives the desired cycle in $G$.

Hence, we may assume that $G-y$ is not 3 -connected. By Theorem 2.1, we decompose $G-y$ into 3-connected components. Let $\mathcal{H}:=H_{1} \ldots H_{h}$ be a block chain in $G-y$ such that (a) $f \in E\left(H_{1}\right)$ and $x \in V\left(H_{h}\right)$, (b) if $h=1$ and $H_{1}=C_{1} \ldots C_{k}$ is a cycle chain with $k \geq 2$, then $x \in V\left(C_{k}\right)-V\left(C_{k-1}\right), f \in E\left(C_{1}\right)$, and $f$ is not incident with both vertices in $V\left(C_{1} \cap C_{2}\right)$, and (c) if $h \geq 2$, then $x \in V\left(H_{h}\right)-V\left(H_{h-1}\right)$, if $H_{h}=C_{1} \ldots C_{k}$ is a cycle chain with $k \geq 2$ and $V\left(H_{h-1} \cap H_{h}\right)=V\left(C_{1} \cap H_{h-1}\right)$, then $x \in V\left(C_{k}\right)-V\left(C_{k-1}\right), f \in E\left(H_{1}\right), f$ is not incident with both vertices in $V\left(H_{1} \cap H_{2}\right)$, and if $H_{1}=C_{1} \ldots C_{k}$ is a cycle chain with $k \geq 2$ and $V\left(H_{1} \cap H_{2}\right)=V\left(C_{k} \cap H_{2}\right)$, then $f \in E\left(C_{1}\right)$ and $f$ is not incident with both vertices in $V\left(C_{1} \cap C_{2}\right)$. Define $V\left(H_{s} \cap H_{s+1}\right)=\left\{a_{s}, b_{s}\right\}$ for $1 \leq s \leq h-1$.

Suppose $V(\mathcal{H})=V(G-y)$. If $h=1$, then $G-y$ is a cycle chain, and it is easy to see that $G$ has a Hamilton cycle through $e$ and $f$, and hence, Theorem 1.2(b) holds. So assume $h \geq 2$. Let $x^{\prime} \in V\left(H_{1}\right)-V\left(H_{2}\right)$ so that $y x^{\prime} \in E(G)$, and in addition, if $f$ has an end with degree 2 in $\mathcal{H}$, then choose $x^{\prime}$ to be that end (in this case, $y x^{\prime} \in E(G)$ ). 
Let $G^{\prime}$ be obtained from $G-y$ by adding $x x^{\prime}$ and then suppressing all degree 2 vertices and deleting separating edges of $\mathcal{H}$. Now $G^{\prime}$ is 3-connected, $\left|G^{\prime}\right| \geq n-1-(d-2)$ (because degree of $y$ in $G$ is at most $d$ ), and $\Delta\left(G^{\prime}\right) \leq d$. Therefore, by Theorem 1.2(b), $G^{\prime}$ has a cycle $C^{\prime}$ through $f$ and $x x^{\prime}$ such that $\left|C^{\prime}\right| \geq \frac{1}{2}\left(\frac{\left|G^{\prime}\right|}{d}\right)^{r}+3$. By replacing edges in $\left(C^{\prime}-x x^{\prime}\right)+\left\{y, y x, y x^{\prime}\right\}$ but not in $G$ with appropriate paths in $G$, we obtain a cycle $C$ in $G$ through $e$ and $f$ such that $|C| \geq\left|C^{\prime}\right|+1 \geq \frac{1}{2}\left(\frac{n-d+1}{d}\right)^{r}+4 \geq \frac{1}{2}\left(\frac{n}{d}\right)^{r}+3$, where the final inequality follows from Lemma 3.1. So $C$ is the desired cycle.

We thus may assume that $\mathcal{H} \neq G-y$. Then there is a 2-cut $\{p, q\}$ of $G-y$ such that $p q$ is a virtual edge in $H_{t}$ for some $1 \leq t \leq h$. Define $G_{1}$ as the graph obtained from $G$ by deleting those components of $(G-y)-\{p, q\}$ containing a vertex of $\mathcal{H}$. Note that $G_{1}-\{p, q, y\}$ contains a neighbor of $y$. We choose $\{p, q\}$ so that $\left|G_{1}\right|$ is maximum. Because $y$ has degree at most $d$ in $G$ and $y x \in E(G)$, and since all degree 2 vertices of $G-y$ are neighbors of $y$, we have (from the choice of $G_{1}$ ) the following observation.

Observation 1. $\left|G_{1}\right| \geq \frac{n-\sigma(\mathcal{H})}{d-1}$.

If there is a 2 -cut $\{v, w\}$ of $G-y$ such that $\{v, w\} \subseteq V\left(\mathcal{H} \cup G_{1}\right)$ and $(G-y)-\{v, w\}$ has a component not containing any vertex of $\mathcal{H} \cup G_{1}$, then let $G_{2}$ denote the graph obtained from $G$ by deleting those components of $(G-y)-\{v, w\}$ containing a vertex of $\mathcal{H} \cup G_{1}$. If such a 2-cut does not exist, then let $G_{2}=\emptyset$. From the definition of $G_{1}$, we see that $\{v, w\} \subseteq V(\mathcal{H}),\{v, w\} \neq\{p, q\}$, and $V\left(G_{1} \cap G_{2}\right) \subseteq\{p, q, y\} \cap\{v, w, y\}$. Choose $\{v, w\}$ so that $\left|G_{2}\right|$ is maximum. By the same reason for Observation 1 , we have the following two observations.

Observation 2. If $\sigma(\mathcal{H}) \geq\left|G_{2}\right|$, then $\sigma(\mathcal{H}) \geq \frac{n-\left|G_{1}\right|}{d-1}$.

Observation 3. If $\left|G_{2}\right| \geq \sigma(\mathcal{H})$, then $\left|G_{2}\right| \geq \frac{n-\left|G_{1}\right|}{d-1}$.

We consider three cases.

Case 1. $\sigma(\mathcal{H}) \geq\left|G_{2}\right|$.

We use $\mathcal{H}$ and $G_{1}$ to find the desired cycle. Choose $t$ so that $\{p, q\} \neq\left\{a_{t}, b_{t}\right\}$. (Note that $a_{t}, b_{t}$ are not defined when $t=h$.) Clearly, $\left|H_{t}\right| \leq n-3$ when $h \geq 2$. By Lemma 3.7(ii), there is a path $P$ from $x$ to $\{p, q\}$ in $\mathcal{H}$ such that $f \in E(P), p q \notin E(P)$ unless $p q=f$, and $|E(P)| \geq \frac{1}{2}\left(\frac{\sigma(\mathcal{H})}{d}\right)^{r}+1$. Assume the notation of $\{p, q\}$ is chosen so that $P$ is from $x$ to $p$.

Since $G$ is 3 -connected, $G_{1}^{\prime}:=G_{1}+\{y p, y q, p q\}$ is 3-connected. If $G_{1}^{\prime} \cong K_{4}$, then we can find a path $Q$ in $G_{1}^{\prime}-q$ from $p$ to $y$ such that $|E(Q)|=2 \geq \frac{1}{2}\left(\frac{\left|G_{1}\right|}{d}\right)^{r}+1$. Now assume that $G_{1}^{\prime} \neq K_{4}$. Note that $\Delta\left(G_{1}^{\prime}\right) \leq d+1$, and $y, p, q$ are the only possible vertices with degree $d+1$. By Theorem $1.2(\overline{\mathrm{a}})$, there is a cycle $C_{1}$ through $p y$ in $G_{1}^{\prime}-q$ such that $\left|C_{1}\right| \geq \frac{1}{2}\left(\frac{(d-1)\left|G_{1}\right|}{d t_{1}}\right)^{r}+2$, where $t_{1} \leq d-1$ is the number of neighbors of $q$ in $G_{1}^{\prime}$ distinct from $p$ and $y$. Hence, $\left|C_{1}\right| \geq \frac{1}{2}\left(\frac{\left|G_{1}\right|}{d}\right)^{r}+2$.

Let $C^{*}:=\left(P \cup\left(C_{1}-p y\right)\right)+x y$. Then $C^{*}$ is a cycle through $e$ and $f$ and $\left|C^{*}\right| \geq \frac{1}{2}\left[\left(\frac{\sigma(\mathcal{H})}{d}\right)^{r}+\left(\frac{\left|G_{1}\right|}{d}\right)^{r}\right]+3$. If $\sigma(\mathcal{H}) \leq\left|G_{1}\right|$, then

$$
\begin{aligned}
\left|C^{*}\right| & \geq \frac{1}{2}\left(\frac{(b-1) \sigma(\mathcal{H})}{d}+\frac{\left|G_{1}\right|}{d}\right)^{r}+3 \quad \text { (by Lemma 3.1) } \\
& >\frac{1}{2}\left(\frac{n-\left|G_{1}\right|}{d}+\frac{\left|G_{1}\right|}{d}\right)^{r}+3 \quad(\text { by Observation 2) } \\
& =\frac{1}{2}\left(\frac{n}{d}\right)^{r}+3 .
\end{aligned}
$$


So we may assume $\sigma(\mathcal{H}) \geq\left|G_{1}\right|$. Then

$$
\begin{aligned}
\left|C^{*}\right| & \geq \frac{1}{2}\left(\frac{\sigma(\mathcal{H})}{d}+\frac{(b-1)\left|G_{1}\right|}{d}\right)^{r}+3 \quad(\text { by Lemma 3.1) } \\
& >\frac{1}{2}\left(\frac{\sigma(\mathcal{H})}{d}+\frac{n-\sigma(\mathcal{H})}{d}\right)^{r}+3 \quad(\text { by Observation } 1) \\
& =\frac{1}{2}\left(\frac{n}{d}\right)^{r}+3 .
\end{aligned}
$$

The desired cycle $C$ can be obtained from $C^{*}$ by replacing virtual edges in $C^{*}$ with appropriate paths in $G$.

Case 2. $\sigma(\mathcal{H})<\left|G_{2}\right|$.

Then $G_{2}$ is nonempty. We use $G_{1}$ and $G_{2}$ to find the desired cycle. There exists some $1 \leq u \leq h$ such that $\{v, w\} \subseteq V\left(H_{u}\right)$, and we may choose $u$ so that $\{v, w\} \neq\left\{a_{u-1}, b_{u-1}\right\}$. (Note that $a_{u-1}, b_{u-1}$ are not defined when $u=1$.) We may choose $t$ so that $\{p, q\} \neq\left\{a_{t-1}, b_{t-1}\right\}$. Again, $a_{t-1}, b_{t-1}$ are not defined when $t=1$.

(1) We claim that there is a path $P$ in $\mathcal{H}$ from $x$ to some $z \in\{p, q\} \cup\{v, w\}$ and containing no separating edge of $\mathcal{H}$ such that (i) $f \in E(P)$, (ii) $p q \in E(P)$ or $v w \in E(P)$, (iii) if $p q \in E(P)$, then $z \in\{v, w\}$, and $v w \notin E(P)$ unless $v w=f$, and (iv) if $v w \in E(P)$, then $z \in\{p, q\}$, and $p q \notin E(P)$ unless $p q=f$.

We prove (1) for $t \leq u$; the case $t \geq u$ can be treated in the same way.

First, we define $Q$. When $t \neq 1$, we find a cycle $Q^{\prime}$ in $\bigcup_{s=1}^{t-1} H_{s}$ through $a_{t-1} b_{t-1}$ and $f$ and containing no separating edge of $\mathcal{H}$ (except $a_{t-1} b_{t-1}$ ). Let $Q:=Q^{\prime}-$ $a_{t-1} b_{t-1}$, which is a path from $a_{t-1}$ to $b_{t-1}$ through $f$. Let $Q=\emptyset$ when $t=1$.

Suppose $t<u$. Since removing separating edges of $H_{t+1} \ldots H_{h}$ that are different from $v w$ results in a 2-connected graph, we may choose the notation of $\left\{a_{t}, b_{t}\right\}$ so that $\left(\bigcup_{s=t+1}^{h} H_{s}\right)-b_{t}$ contains a path $X$ from $a_{t}$ to $x$ through $v w$ and containing no separating edge of $\mathcal{H}$ (except possibly $v w$ ).

We claim that there is a path $C_{t}$ in $H_{t}-a_{t} b_{t}$ from $a_{t}$ to $\{p, q\}$ through $a_{t-1} b_{t-1}$ (or $f$ when $t=1$ ), or a path $C_{t}^{\prime}$ in $H_{t}$ from $a_{t}$ to $b_{t}$ through $a_{t-1} b_{t-1}$ (or $f$ when $t=1$ ) and $p q$. If $\{p, q\}=\left\{a_{t}, b_{t}\right\}$, then the existence of $C_{t}$ follows from 2-connectivity of $H_{t}$. So we may assume that $\{p, q\} \neq\left\{a_{t}, b_{t}\right\}$. Again by 2-connectivity of $H_{t}$ there is a cycle $D$ in $H_{t}$ through $p q$ and $a_{t-1} b_{t-1}$ (or $f$ when $t=1$ ). If $a_{t} b_{t} \in E(D)$, then $C_{t}^{\prime}:=D-a_{t} b_{t}$ is as desired. So we may assume $a_{t} b_{t} \notin E(D)$. By 2-connectivity of $H_{t}$, there is a path $A$ in $H_{t}$ from $a_{t}$ to $D$ and internally disjoint from $D$. One can easily check that $C_{t}$ exists in $A \cup D$.

If we find $C_{t}$, then let $P_{t}:=C_{t}-a_{t-1} b_{t-1}$ when $t \neq 1$ and $P_{t}:=C_{t}$ when $t=1$. In this case, $P:=Q \cup P_{t} \cup X$ gives the desired path for (1). So assume that we find $C_{t}^{\prime}$. Let $P_{t}:=C_{t}^{\prime}$ if $t=1$, and otherwise let $P_{t}:=C_{t}^{\prime}-a_{t-1} b_{t-1}$. Let $H:=H_{t+1} \ldots H_{h}$. If $x \in\{v, w\}$, then we find a cycle $C^{\prime}$ in $H$ through $a_{t} b_{t}$ and $v w$ and containing no separating edge of $\mathcal{H}$ (except $a_{t} b_{t}$ and $v w$ ), and $P:=Q \cup P_{t} \cup\left(C^{\prime}-\left\{a_{t} b_{t}, v w\right\}\right.$ ) gives the desired path for (1). Therefore, we may assume $x \notin\{v, w\}$. Let $H^{\prime}$ be obtained from $H$ by a T-transform at $\{x, v w\}$, let $x^{\prime}$ denote the new vertex, and let $H^{\prime \prime}$ be obtained from $H^{\prime}$ by deleting all separating edges of $H$ different from $a_{t} b_{t}$. Then $H^{\prime \prime}$ is a 2-connected graph. So there is a cycle $C^{\prime \prime}$ in $H^{\prime \prime}$ through $a_{t} b_{t}$ and $x x^{\prime}$. Now $P:=Q \cup P_{t} \cup\left(C^{\prime \prime}-\left\{x^{\prime}, a_{t} b_{t}\right\}\right)$ gives the desired path for (1).

Therefore, we may assume $t=u$. We claim that there is a path $Q_{t}$ in $H_{t}$ from $\left\{a_{t}, b_{t}\right\}$ when $t \neq h$, or from $x$ when $t=h$, to some $z \in\{p, q\} \cup\{v, w\}$ such that (i) $a_{t-1} b_{t-1} \in E\left(Q_{t}\right)$ (or $f \in E\left(Q_{t}\right)$ when $t=1$ ), (ii) $p q \in E\left(Q_{t}\right)$ or $v w \in E\left(Q_{t}\right)$, (iii) if $p q \in E\left(Q_{t}\right)$, then $z \in\{v, w\}$, and $v w \notin E\left(Q_{t}\right)$ unless $v w=f$, and (iv) if $v w \in E\left(Q_{t}\right)$, 
then $z \in\{p, q\}$, and $p q \notin E\left(Q_{t}\right)$ unless $p q=f$. This is easy to see if $H_{t}$ is a cycle chain (because $p q \neq v w$ ). Otherwise, it follows from Lemma 2.8 or Lemma 2.9 when $f \notin\{p q, v w\}$, and follows from 3-connectivity of $H_{t}$ when $f \in\{p q, v w\}$.

Assume without loss of generality that $a_{t}$ is an end of $Q_{t}$. When $t \neq h$, we find a path $R$ from $a_{t}$ to $x$ in $\left(H_{t+1} \ldots H_{h}\right)-b_{t}$ containing no separating edge of $\mathcal{H}$. When $t=h$, let $R=\emptyset$. Let $P_{t}:=Q_{t}$ when $t=1$, and otherwise let $P_{t}:=Q_{t}-a_{t-1} b_{t-1}$. Then $P:=Q \cup P_{t} \cup R$ gives the desired path for (1).

We may assume that $v w \in E(P)$ and $p$ is an end of $P$, since the case $p q \in E(P)$ is similar.

(2) Note that $G_{1}^{\prime}:=G_{1}+\{y p, y q, p q\}$ is 3-connected, $\Delta\left(G_{1}^{\prime}\right) \leq d+1$, and $y, p, q$ are the possible vertices of degree $d+1$ in $G_{1}^{\prime}$. If $G_{1}^{\prime} \cong K_{4}$, then we can find a path $P_{1}$ from $p$ to $y$ in $G_{1}^{\prime}-q$ such that $\left|E\left(P_{1}\right)\right|=2 \geq \frac{1}{2}\left(\frac{\left|G_{1}\right|}{d}\right)^{r}+1$. If $G_{1}^{\prime} \neq K_{4}$, then by Theorem 1.2(a), there is a cycle $C_{1}$ through $p y$ in $G_{1}^{\prime}-q$ such that $\left|C_{1}\right| \geq$ $\frac{1}{2}\left(\frac{(d-1)\left|G_{1}\right|}{d t_{1}}\right)^{r}+2$, where $t_{1} \leq d-1$ is the number of neighbors of $q$ in $G_{1}^{\prime}$ distinct from $p$ and $y$. Let $P_{1}:=C_{1}-p y$; then $\left|E\left(P_{1}\right)\right| \geq \frac{1}{2}\left(\frac{\left|G_{1}\right|}{d}\right)^{r}+1$.

(3) Note that $G_{2}^{\prime}:=G_{2}+\{y v, y w, v w\}$ is 3-connected, $\Delta\left(G_{2}^{\prime}\right) \leq d+1$, and $y, v, w$ are the possible vertices of degree $d+1$ in $G_{2}^{\prime}$. If $G_{2}^{\prime} \cong K_{4}$, then we can find a path $P_{2}$ from $v$ to $w$ in $G_{2}^{\prime}-y$ such that $\left|E\left(P_{2}\right)\right|=2 \geq \frac{1}{2}\left(\frac{\left|G_{2}\right|}{d}\right)^{r}+1$. If $G_{2}^{\prime} \neq K_{4}$, then by Theorem 1.2(a), there is a cycle $C_{2}$ through $v w$ in $G_{2}^{\prime}-y$ such that $\left|C_{2}\right| \geq \frac{1}{2}\left(\frac{(d-1)\left|G_{2}^{\prime}\right|}{d t_{2}}\right)^{r}+2$, where $t_{2} \leq d-1$ is the number of neighbors of $y$ in $G_{2}^{\prime}$ distinct from $v$ and $w$. Let $P_{2}:=C_{2}-v w$; then $\left|E\left(P_{2}\right)\right| \geq \frac{1}{2}\left(\frac{\left|G_{2}\right|}{d}\right)^{r}+1$.

Let $C^{*}:=\left((P-v w) \cup P_{1} \cup P_{2}\right)+e$. Then $C^{*}$ is a cycle through $e$ and $f$ and

$$
\begin{aligned}
\left|C^{*}\right| & \geq\left|E\left(P_{1}\right)\right|+\left|E\left(P_{2}\right)\right|+1 \\
& \geq \frac{1}{2}\left(\frac{\left|G_{1}\right|}{d}\right)^{r}+\frac{1}{2}\left(\frac{\left|G_{2}\right|}{d}\right)^{r}+3 \quad \text { (by (2) and (3)) } \\
& \left.\geq \frac{1}{2}\left(\frac{\left|G_{1}\right|}{d}+\frac{(b-1)\left|G_{2}\right|}{d}\right)^{r}+3 \quad \text { (by Lemma } 3.1 \text { and since }\left|G_{1}\right| \geq\left|G_{2}\right|\right) \\
& \left.>\frac{1}{2}\left(\frac{\left|G_{1}\right|}{d}+\frac{n-\left|G_{1}\right|}{d}\right)^{r}+3 \quad \text { (by Observation } 3 \text { and since }\left|G_{2}\right| \geq \sigma(\mathcal{H})\right) \\
& =\frac{1}{2}\left(\frac{n}{d}\right)^{r}+3 .
\end{aligned}
$$

As before, the desired cycle $C$ can be obtained by modifying $C^{*}$.

5. Cycles through one edge. We now reduce Theorem 1.2(c); our proof implies an $O(E)$ time reduction. Here we use Lemmas 3.2 and 3.3, and need $b=\max \{64,4 d+1\}$.

Lemma 5.1. Let $n \geq 6$ be an integer, and assume that Theorem 1.2 holds for graphs with at most $n-1$ vertices. Let $G$ be a 3 -connected graph with $n$ vertices and $\Delta(G) \leq d$. Then for any $e \in E(G)$, there is a cycle $C$ through $e$ in $G$ such that $|C| \geq \frac{1}{2} n^{r}+3$.

Proof. By Lemma 1.3, we may assume $n>(4 d+1)^{2}$. Let $e=x y \in E(G)$. If $G-y$ is 3-connected, then let $y^{\prime}$ be a neighbor of $y$ other than $x$. Clearly, $G^{\prime}:=(G-y)+x y^{\prime}$ is 3-connected, $\Delta\left(G^{\prime}\right) \leq d$, and $5 \leq\left|G^{\prime}\right|<n$. By Theorem 1.2(c), there is a cycle $C^{\prime}$ through $x y^{\prime}$ in $G^{\prime}$ such that $\left|C^{\prime}\right| \geq \frac{1}{2}(n-1)^{r}+3$. Now let $C:=\left(C^{\prime}-x y^{\prime}\right)+\left\{y, x y, y y^{\prime}\right\}$. Then $C$ is a cycle through $x y$ in $G$ and, by Lemma 3.1,

$$
|C|=\left|C^{\prime}\right|+1 \geq \frac{1}{2}(n-1)^{r}+1+3 \geq \frac{1}{2} n^{r}+3 .
$$


Therefore, we may assume that $G-y$ is not 3 -connected. Since $G-y$ is 2connected, we use Theorem 2.1 to decompose $G-y$ into 3-connected components.

Suppose all 3-blocks of $G-y$ are cycles. Let $\mathcal{L}=L_{1} \ldots L_{\ell}$ be a cycle chain in $G-y$ such that (i) $x \in V\left(L_{1}\right)$, (ii) $L_{\ell}$ is an extreme 3-block of $G-y$, and (iii) subject to (i) and (ii), $|\mathcal{L}|$ is maximum. Because $G$ is 3 -connected, each degree 2 vertex in $\mathcal{L}$ is a neighbor of $y$ or is contained in a 3 -block of $G-y$ not in $\mathcal{L}$. Hence, it is easy to see that there is some $y^{\prime} \in V(\mathcal{L})-\{x\}$ such that $\mathcal{L}$ contains a Hamilton path $P$ from $x$ to $y^{\prime}$ and $G$ has a path $Q$ from $y^{\prime}$ to $y$ disjoint from $V(\mathcal{L})-\left\{y^{\prime}\right\}$. Let $C:=(P \cup Q)+\left\{y, x y, y y^{\prime}\right\}$, which is a cycle in $G$. Then $|C| \geq|\mathcal{L}|+1$. If $V(G-y)=V(\mathcal{L})$, then $|C|=n \geq \frac{1}{2} n^{r}+3$ (since $n \geq 5$ and by Lemma 3.4). So we may assume $V(G-y) \neq V(\mathcal{L})$. Write $B:=L_{1}$. Because $x \in V\left(L_{1}\right)$ and $x y \in E(G)$, it follows from (iii) that $|\mathcal{L}| \geq \frac{(n-1)-|B|}{t}+|B|=\frac{n+(t-1)|B|-1}{t}$, where $t$ is the number of extreme 3-blocks of $G-y$ distinct from $L_{1}$. So $2 \leq t \leq d-1$ (because $V(G-y) \neq V(\mathcal{L})$ ). Then $|C| \geq|\mathcal{L}|+1 \geq \frac{n+(t-1)|B|-1}{t}+1$. Note that $|C|-3 \geq \frac{n+(t-1)|B|-1}{t}-2 \geq \frac{n+t-4}{t}$ (since $|B| \geq 3$ ). Using elementary calculus, we can show that the function $\frac{x+t-t}{t}-\frac{1}{2} x^{r}$ is increasing when $x \geq(4 d+1)^{2}$. Hence $\frac{n+t-4}{t} \geq \frac{1}{2} n^{r}$ (because $t \leq d-1$ and $\left.n \geq(4 d+1)^{2}\right)$. Therefore, $|C| \geq \frac{1}{2} n^{r}+3$ and $C$ gives the desired cycle in $G$.

Hence, we may assume that not all 3 -blocks of $G-y$ are cycles. We choose a 3-connected 3-block $H_{0}$ of $G-y$ with $\left|H_{0}\right|$ maximum. Let $\mathcal{H}=H_{0} H_{1} H_{2} \ldots H_{h}$ be a block chain in $G-y$ such that either $h=0$ and $x \in V\left(H_{0}\right)$, or $h \geq 1$ and $x \in V\left(H_{h}\right)-V\left(H_{h-1}\right)$, and if $H_{h}=C_{1} \ldots C_{k}$ is a cycle chain with $k \geq 2$ and $V\left(H_{h-1} \cap H_{h}\right)=V\left(C_{1} \cap C_{2}\right)$, then $x \in V\left(C_{k}\right)-V\left(C_{k-1}\right)$. For $0 \leq i \leq h-1$, let $V\left(H_{i} \cap H_{i+1}\right)=\left\{a_{i}, b_{i}\right\}$.

If $V(G-y) \neq V(\mathcal{H})$, there is a block chain $\mathcal{L}:=L_{1} L_{2} \ldots L_{\ell}$ in $G-y$ such that $V(\mathcal{H} \cap \mathcal{L})=V\left(\mathcal{H} \cap L_{1}\right)$ consists of two vertices $c_{0}$ and $d_{0}, L_{\ell}$ is (or contains) an extreme 3-block of $G-y$, and if $L_{1}=C_{1} \ldots C_{k}$ is a cycle chain with $k \geq 2$ and $V\left(L_{1} \cap L_{2}\right)=V\left(C_{k} \cap H_{2}\right)$ when $\ell \geq 2$, then $c_{0} d_{0} \in E\left(C_{1}\right)$ and $\left\{c_{0}, d_{0}\right\} \neq V\left(C_{1} \cap C_{2}\right)$. For $1 \leq i \leq \ell-1$, let $V\left(L_{i} \cap L_{i+1}\right)=\left\{c_{i}, d_{i}\right\}$. If $\mathcal{L}$ exists, we choose $\mathcal{L}$ so that $\sigma(\mathcal{L})$ is maximum.

(1) We may assume $V(G-y) \neq V(\mathcal{H})$, and $\sigma(\mathcal{L})+2 \geq \frac{n-\sigma(\mathcal{H})-1}{d-1}$.

Suppose $V(G-y)=V(\mathcal{H})$. When $h=0$, let $x^{\prime}$ be a neighbor of $y$ in $H_{0}-x$, otherwise, let $x^{\prime}$ be a neighbor of $y$ in $H_{0}-V\left(H_{1}\right)$. Let $G^{\prime}$ be obtained from $H+x x^{\prime}$ by suppressing all degree 2 vertices and deleting separating edges of $\mathcal{H}$. Then $G^{\prime}$ is 3 -connected. By Theorem 1.2(c), there is a cycle $C^{\prime}$ in $G^{\prime}$ through $x x^{\prime}$ such that $\left|C^{\prime}\right| \geq \frac{1}{2}\left|G^{\prime}\right|^{r}+3$. Let $C^{*}:=\left(C^{\prime}-x x^{\prime}\right)+\left\{y, y x, y x^{\prime}\right\}$. Since $\Delta(G) \leq d,\left|G^{\prime}\right| \geq$ $(n-1)-(d-2)$. Hence, $\left|C^{*}\right|=\left|C^{\prime}\right|+1 \geq \frac{1}{2}(n-d+1)^{r}+1+3>\frac{1}{2} n^{r}+3$ (by Lemma 3.1). Clearly, the desired cycle $C$ can be obtained by modifying $C^{*}$.

So we may assume $V(G-y) \neq V(\mathcal{H})$. Note that any vertex of $G$ not contained in any $A\left(H_{i}\right), 1 \leq i \leq h$, either is counted in $\sigma\left(\mathcal{L}^{\prime}\right)+2$ for some block chain $\mathcal{L}^{\prime}$ defined as $\mathcal{L}$ except the maximum requirement (the constant 2 counts the vertices in $V\left(\mathcal{H} \cap \mathcal{L}^{\prime}\right)$ ), or is a degree 2 vertex in $G-y$ (and hence a neighbor of $y$ ). Therefore, since $x y \in E(G)$ and $\Delta(G) \leq d, \sigma(\mathcal{L})+2 \geq \frac{n-\sigma(\mathcal{H})-1}{d-1}$.

(2) There exists a path $P$ in $\mathcal{H}$ from $x$ to $\left\{c_{0}, d_{0}\right\}$ such that $c_{0} d_{0} \notin E(P)$ and $|E(P)| \geq \frac{1}{2}\left|H_{0}\right|^{r}+\frac{1}{2}\left(\sum\left\{\left(\frac{\left|H_{i}\right|}{d}\right)^{r}: i \neq 0\right.\right.$ and $\left.\left.H_{i} \in B_{1}(\mathcal{H})\right\}\right)+\left(\sum\left\{\max \left\{1,\left|A\left(H_{i}\right)\right|-2\right\}:\right.\right.$ $i \neq 0$ and $\left.\left.H_{i} \in B_{1}(\mathcal{H})\right\}\right)+1$. In particular, $|E(P)| \geq \frac{1}{2}(\sigma(\mathcal{H}))^{r}+1$.

The first part of (2) follows from Lemma 3.7(i). The second part of (2) follows from Lemma 3.1. When applying Lemma 3.1, we express $\max \left\{1,\left|A\left(H_{i}\right)\right|-2\right\}$ as the sum of 1 , and we use $b \geq 4 d+1,(b-1)\left(\left|A\left(H_{i}\right)\right|-2\right) \geq\left|A\left(H_{i}\right)\right|$ when $\left|A\left(H_{i}\right)\right| \geq 3$, 
and the fact that $\left|H_{0}\right| \geq\left|H_{i}\right|$ for all 3-connected $H_{i}$.

(3) We may assume $\sigma(\mathcal{H})<\frac{n-1}{4}$.

Suppose $\sigma(\mathcal{H}) \geq \frac{n-1}{4}$. Without loss of generality, assume $c_{0}$ is an end of the path $P$ in (2). By Lemma 3.6(i), there is a path $Q$ in $\mathcal{L}-d_{0}$ from $c_{0}$ to some $y^{\prime} \in N(y) \cap V\left(L_{\ell}\right)$ such that $|E(Q)| \geq \frac{1}{2}\left(\frac{\sigma(\mathcal{L})}{d}\right)^{r}+1$. Let $C^{*}:=(P \cup Q)+\left\{y, y y^{\prime}, y x\right\}$. Then

$$
\left|C^{*}\right|=|E(P)|+|E(Q)|+2 \geq \frac{1}{2}(\sigma(\mathcal{H}))^{r}+1+\frac{1}{2}\left(\frac{\sigma(\mathcal{L})}{d}\right)^{r}+3 .
$$

If $\sigma(\mathcal{H}) \leq \frac{b(b-1)}{4} \frac{\sigma(\mathcal{L})}{d}$, then by Lemmas 3.3 and 3.1,

$$
\left|C^{*}\right| \geq \frac{1}{2}(4 \sigma(\mathcal{H})+1)^{r}+3 \geq \frac{1}{2} n^{r}+3 .
$$

If $\sigma(\mathcal{H}) \geq \frac{b(b-1)}{4} \frac{\sigma(\mathcal{L})}{d}$, then by Lemma 3.2 and since $b \geq 4 d+1$,

$$
\begin{aligned}
\left|C^{*}\right| & >\frac{1}{2}\left(\sigma(\mathcal{H})+\frac{b(b-1)}{4} \frac{\sigma(\mathcal{L})}{d}+2(b-1)\right)^{r}+3 \\
& \geq \frac{1}{2}(\sigma(\mathcal{H})+(4 d+1) \sigma(\mathcal{L})+8 d)^{r}+3 \\
& >\frac{1}{2} n^{r}+3 .
\end{aligned}
$$

The final inequality holds by $(1)$ and $\sigma(\mathcal{H})<n-1$. Now the desired cycle $C$ can be obtained from $C^{*}$ by replacing virtual edges in $C^{*}$ with appropriate paths in $G$.

(4) We may assume $\left|H_{0}\right|+4\left(\sigma(\mathcal{H})-\left|H_{0}\right|+\sigma(\mathcal{L})\right)<n$. In particular, $\sigma(\mathcal{L}) \leq$ $\frac{n-1-\left|H_{0}\right|}{4}$.

Suppose $\left|H_{0}\right|+4\left(\sigma(\mathcal{H})-\left|H_{0}\right|+\sigma(\mathcal{L})\right) \geq n$. Without loss of generality, assume that the path $P$ in (2) is from $x$ to $c_{0}$. By Lemma 3.6(ii), there is a path $Q$ in $\mathcal{L}-d_{0}$ from $c_{0}$ to some $y^{\prime} \in N(y) \cap V\left(L_{\ell}\right)$ such that $|E(Q)| \geq \frac{1}{2}\left(\sum\left\{\left(\frac{\left|A\left(L_{i}\right)\right|}{d}\right)^{r}: L_{i} \in\right.\right.$ $\left.\left.B_{1}(\mathcal{L})\right\}\right)+\left(\sum\left\{\max \left\{1,\left|A\left(L_{i}\right)\right|-2\right\}: L_{i} \in B_{2}(\mathcal{L})\right\}\right)+1$.

Let $C^{*}=(P \cup Q)+\left\{y, y y^{\prime}, y x\right\}$. Then by (2) and above, $\left|C^{*}\right|=|E(P)|+|E(Q)|+$ $2 \geq \frac{1}{2}\left|H_{0}\right|^{r}+\frac{1}{2}\left(\sum\left\{\left(\frac{\left|A\left(H_{i}\right)\right|}{d}\right)^{r}: i \neq 0\right.\right.$ and $\left.\left.H_{i} \in B_{1}(\mathcal{H})\right\}\right)+\left(\sum\left\{\max \left\{1,\left|A\left(H_{i}\right)\right|-2\right\}:\right.\right.$ $i \neq 0$ and $\left.\left.H_{i} \in B_{2}(\mathcal{H})\right\}\right)+\frac{1}{2}\left(\sum\left\{\left(\frac{\left|A\left(L_{i}\right)\right|}{d}\right)^{r}: L_{i} \in B_{1}(\mathcal{L})\right\}\right)+\left(\sum\left\{\max \left\{1,\left|A\left(L_{i}\right)\right|-2\right\}:\right.\right.$ $\left.\left.L_{i} \in B_{2}(\mathcal{L})\right\}\right)+4$. Because $\left|H_{0}\right|$ is maximum among all 3-connected 3-blocks of $G-y$, it follows from Lemma 3.1 and the fact that $b \geq 4 d+1$ that

$$
\begin{aligned}
\left|C^{*}\right| & \geq \frac{1}{2}\left[\left|H_{0}\right|+4\left(\sum_{i=1}^{h}\left|A\left(H_{i}\right)\right|+\sum_{j=1}^{\ell}\left|A\left(L_{j}\right)\right|\right)\right]^{r}+4 \\
& =\frac{1}{2}\left[\left|H_{0}\right|+4\left(\sigma(\mathcal{H})-\left|H_{0}\right|+\sigma(\mathcal{L})\right)\right]^{r}+4 \\
& >\frac{1}{2} n^{r}+3 .
\end{aligned}
$$

As before, the desired cycle $C$ can be obtained by modifying $C^{*}$. This proves (4).

We need to consider block chains other than $\mathcal{H}$ and $\mathcal{L}$. A block chain $\mathcal{M}:=$ $M_{1} M_{2} \ldots M_{m}$ is called an $\mathcal{H} \mathcal{L}$-leg if $M_{m}$ contains an extreme 3-block of $G-y$ and $V(\mathcal{M} \cap(\mathcal{H} \cup \mathcal{L}))$ consists of two vertices $x_{0}$ and $y_{0}$ such that $\left\{x_{0}, y_{0}\right\} \subseteq V\left(M_{1}\right)$ and $\left\{x_{0}, y_{0}\right\} \neq V\left(M_{1} \cap M_{2}\right)$ when $m \geq 2$, and if $M_{1}=C_{1} \ldots C_{k}$ is a cycle chain with 
$k \geq 2$ and $V\left(C_{k} \cap M_{2}\right)=V\left(M_{1} \cap M_{2}\right)$ when $m \geq 2$, then $\left\{x_{0}, y_{0}\right\} \subseteq V\left(C_{1}\right)$ and $\left\{x_{0}, y_{0}\right\} \neq V\left(C_{1} \cap C_{2}\right)$. We view degree 2 vertices of $G-y$ (which are neighbors of $y$ ) as trivial $\mathcal{H} \mathcal{L}$-legs.

(5) We may assume that there is an $\mathcal{H} \mathcal{L}$-leg $\mathcal{M}$ such that $\sigma(\mathcal{M})>\frac{n}{4(d-2)}>4 d+2$.

Note that each extreme 3-block of $G-y$ contains a neighbor of $y$. Since $\Delta(G) \leq d$, there are at most $d-2 \mathcal{H} \mathcal{L}$-legs in $G-y$ (including those trivial ones). Choose an $\mathcal{H} \mathcal{L}$-leg $\mathcal{M}$ such that $\sigma(\mathcal{M})$ is maximum. Note that every vertex of $G-y$ is either a degree 2 vertex (hence covered in a trivial $\mathcal{H} \mathcal{L}$-leg), or is counted in $\sigma(\mathcal{H})$, or in $\sigma(\mathcal{L})+2$, or in $\sigma(\mathcal{M})+2$ for some $\mathcal{H} \mathcal{L}$-leg $\mathcal{M}$. Hence, because $\sigma(\mathcal{H})<\frac{n-1}{4}$ (by (3)) and $\sigma(\mathcal{L}) \leq \frac{n-1-\left|H_{0}\right|}{4} \leq \frac{n-5}{4}$ (by (4) and $\left.\left|H_{0}\right| \geq 4\right), \sigma(\mathcal{M})+2 \geq \frac{n-1-\sigma(\mathcal{H})-\sigma(\mathcal{L})-2}{d-2}>$ $\frac{n-3}{2(d-2)}$. Since we assume $n>(4 d+1)^{2}, \sigma(\mathcal{M})>\frac{n}{4(d-2)}>4 d+2$.

Let $\mathcal{M}$ be an $\mathcal{H} \mathcal{L}$-leg in $G-y$ with $\sigma(\mathcal{M}) \geq \frac{n}{4(d-2)}$. By $(5), \mathcal{M}$ is nontrivial. Let $x_{0}$ and $y_{0}$ be the vertices in $V(\mathcal{M} \cap(\mathcal{H} \cup \mathcal{L}))$. We consider three cases.

Case 1 . $\mathcal{M}$ may be chosen so that $x \notin\left\{x_{0}, y_{0}\right\} \cap\left\{c_{0}, d_{0}\right\}$ and $\left\{x_{0}, y_{0}\right\} \nsubseteq V(\mathcal{H})$.

Then we may assume $\left\{x_{0}, y_{0}\right\} \subseteq V\left(L_{t}\right)$ with $\left\{x_{0}, y_{0}\right\} \neq\left\{c_{t-1}, d_{t-1}\right\}$.

We claim that there is a path $P^{\prime}$ in $\mathcal{H}$ from $x$ to $z \in\left\{c_{0}, d_{0}\right\}$ such that (i) $\left|E\left(P^{\prime}\right)\right| \geq \frac{1}{2}\left(\frac{\left|H_{0}\right|}{d}\right)^{r}+1$, (ii) $c_{0} d_{0} \notin E\left(P^{\prime}\right)$, and (iii) if $z \notin\left\{c_{0}, d_{0}\right\} \cap\left\{x_{0}, y_{0}\right\}$, then $\left\{c_{0}, d_{0}\right\} \cap\left\{x_{0}, y_{0}\right\}=\emptyset$ or $\left\{c_{0}, d_{0}\right\} \cap\left\{x_{0}, y_{0}\right\} \nsubseteq V\left(P^{\prime}\right)$. Choose $z^{\prime} \in\left\{c_{0}, d_{0}\right\}$ such that, if possible, $z^{\prime} \in\left\{c_{0}, d_{0}\right\} \cap\left\{x_{0}, y_{0}\right\}$. Suppose $c_{0} d_{0} \in E\left(H_{1} \ldots H_{h}\right)$. Deleting separating edges of $H_{1} \ldots H_{h}$ results in a 2-connected graph, which contains disjoint paths $Q_{1}, Q_{2}$ from $x, z^{\prime}$ to $a_{0}, b_{0}$, respectively. In $H_{0}$ we use Theorem $1.2(\mathrm{c})$ to find a cycle $C_{0}$ through $a_{0} b_{0}$ such that $\left|C_{0}\right| \geq \frac{1}{2}\left|H_{0}\right|^{r}+3$. If $c_{0} d_{0} \in E\left(Q_{2}\right)$, then $P^{\prime}:=$ $\left(C_{0}-a_{0} b_{0}\right) \cup Q_{1} \cup\left(Q_{2}-z^{\prime}\right)$ gives the desired path; otherwise, $P^{\prime}:=\left(C_{0}-a_{0} b_{0}\right) \cup Q_{1} \cup Q_{2}$ gives the desired path. So we may assume $c_{0} d_{0} \notin E\left(H_{1} \ldots H_{h}\right)$. Suppose $h=0$. We apply Theorem 1.2(a) to find a cycle $C_{0}$ in $\left(H_{0}+\left\{x c_{0}, x d_{0}\right\}\right)-\left(\left\{c_{0}, d_{0}\right\}-\left\{z^{\prime}\right\}\right)$ through $x z^{\prime}$ such that $\left|C_{0}\right| \geq \frac{1}{2}\left(\frac{\left|H_{0}\right|}{d}\right)^{r}+2$; then $P^{\prime}:=C_{0}-x z^{\prime}$ gives the desired path. So let $h \geq 1$. Then $c_{0} d_{0} \in E\left(H_{0}\right)$ and $\left\{c_{0}, d_{0}\right\} \neq\left\{a_{0}, b_{0}\right\}$. Without loss of generality, we may assume $a_{0} \notin\left\{c_{0}, d_{0}\right\}$. Let $Q^{\prime}$ be a path in $\left(H_{1} \ldots H_{h}\right)-b_{0}$ from $x$ to $a_{0}$ and not containing any separating edge of $\mathcal{H}$. If $z^{\prime}=b_{0}$ we use Theorem 1.2(c) to find a cycle $C_{0}$ through $a_{0} b_{0}$ in $H_{0}$ such that $\left|C_{0}\right| \geq \frac{1}{2}\left|H_{0}\right|^{r}+3$, and $P^{\prime}:=\left(C_{0}-a_{0} b_{0}\right) \cup Q^{\prime}$ (when $\left.c_{0} d_{0} \notin E\left(C_{0}\right)\right)$ or $P^{\prime}:=\left(C_{0}-b_{0}\right) \cup Q^{\prime}$ (when $c_{0} d_{0} \in E\left(C_{0}\right)$ ) gives the desired path. So assume $z^{\prime} \neq b_{0}$. If $z^{\prime} \in\left\{c_{0}, d_{0}\right\} \cap\left\{x_{0}, y_{0}\right\}$, then $z^{\prime}$ has at most $d-1$ neighbors in $H_{0}$ (since $\left.\left\{x_{0}, y_{0}\right\} \neq\left\{c_{0}, d_{0}\right\}\right)$, and in $\left(H_{0}+\left\{a_{0} z^{\prime}, z^{\prime} b_{0}\right\}\right)-b_{0}$ we apply Theorem 1.2(a) to find a cycle $C_{0}$ through $a_{0} z^{\prime}$ such that $\left|C_{0}\right| \geq \frac{1}{2}\left(\frac{\left|H_{0}\right|}{d}\right)^{r}+2$; then $P^{\prime}:=\left(C_{0}-a_{0} z^{\prime}\right) \cup Q^{\prime}$ gives the desired path. So we may assume $z^{\prime} \notin\left\{c_{0}, d_{0}\right\} \cap\left\{x_{0}, y_{0}\right\}$. By the choice of $z^{\prime},\left\{c_{0}, d_{0}\right\} \cap\left\{x_{0}, y_{0}\right\}=\emptyset$ (so (iii) is automatic). Let $H_{0}^{\prime}$ be obtained from $H_{0}$ by an H-transform at $\left\{a_{0} b_{0}, c_{0} d_{0}\right\}$, and let $a^{\prime}, x^{\prime}$ denote the new vertices. By applying Theorem 1.2(c) we find a cycle $C_{0}$ through $a^{\prime} x^{\prime}$ in $H_{0}^{\prime}$ such that $\left|C_{0}\right| \geq \frac{1}{2}\left|H_{0}\right|^{r}+3$. Now $C_{0}-\left\{a^{\prime}, x^{\prime}\right\}$ is a path from some $z \in\left\{c_{0}, d_{0}\right\}$ to some $b^{\prime} \in\left\{a_{0}, b_{0}\right\}$. Then $C_{0}-\left\{a^{\prime}, x^{\prime}\right\}$ and a path in $\left(H_{1} \ldots H_{h}\right)-\left(\left\{a_{0}, b_{0}\right\}-\left\{b^{\prime}\right\}\right)$ from $x$ to $b^{\prime}$ (not containing any separating edge of $\mathcal{H})$ gives the desired path $P^{\prime}$.

Without loss of generality, we may assume that $P^{\prime}$ is from $x$ to $c_{0}$. Then $d_{0} \notin$ $V\left(P^{\prime}\right)$ or $d_{0} \notin\left\{x_{0}, y_{0}\right\}$. Therefore, since each $L_{i}$ is 3 -connected or is a cycle chain, there exists a path $Q$ in $\bigcup_{i=0}^{t} L_{i}$ from $c_{0}$ to some $z \in\left\{c_{t}, d_{t}\right\} \cup\left\{x_{0}, y_{0}\right\}$ such that (i) $c_{0} d_{0} \in E(Q) Q$ contains no separating edge of $\mathcal{L}$ except possibly $c_{t} d_{t}$ and $x_{0} y_{0}$, (ii) $Q$ avoids $d_{0}$ if $d_{0} \in V\left(P^{\prime}\right)$ (since in that case $\left\{x_{0}, y_{0}\right\} \cap\left\{c_{0}, d_{0}\right\}=\emptyset$ ), (iii) if $z \in\left\{c_{t}, d_{t}\right\}$, then $x_{0} y_{0} \in E(Q)$, and $c_{t} d_{t} \notin E(Q)$ unless $x_{0} y_{0}=c_{t} d_{t}$, and (iv) if $z \in\left\{x_{0}, y_{0}\right\}$, then $c_{t} d_{t} \in E(Q)$, and $x_{0} y_{0} \notin E(Q)$ unless $x_{0} y_{0}=c_{t} d_{t}$. 
Suppose $z \in\left\{c_{t}, d_{t}\right\}$, and assume the notation is chosen so that $z=c_{t}$. By Lemma 3.6(ii) there is a path $P_{1}$ in $\left(L_{t+1} \ldots L_{\ell}\right)-d_{t}$ from $z$ to some $y^{\prime} \in N(y) \cap$ $V\left(L_{\ell}\right)$ and containing no separating edge of $\mathcal{L}$ such that $\left|E\left(P_{1}\right)\right| \geq \frac{1}{2}\left(\sum\left\{\left(\frac{\left|A\left(L_{i}\right)\right|}{d}\right)^{r}\right.\right.$ : $t+1 \leq i \leq \ell$ and $\left.\left.L_{i} \in B_{1}(\mathcal{L})\right\}\right)+\left(\sum\left\{\max \left\{1,\left|A\left(L_{i}\right)\right|-2\right\}: t+1 \leq i \leq \ell\right.\right.$ and $\left.\left.L_{i} \in B_{2}(\mathcal{L})\right\}\right)+1$. By Lemma 3.5, let $P_{2}$ be a path from $x_{0}$ to $y_{0}$ in $\mathcal{M}$ such that $\left|E\left(P_{2}\right)\right| \geq \frac{1}{2}\left(\frac{(d-1) \sigma(\mathcal{M})}{d}\right)^{r}+1$. Let $n^{*}:=\sum_{i=t+1}^{\ell}\left|A\left(L_{i}\right)\right|$; then by the choice of $\mathcal{L}$, $n^{*} \geq \sigma(\mathcal{M})-2$. Let $C^{*}:=\left(P^{\prime} \cup\left(Q-x_{0} y_{0}\right) \cup P_{1} \cup P_{2}\right)+\left\{y, y y^{\prime}, y x\right\}$. As in the proof of $(4)$,

$$
\begin{aligned}
\left|C^{*}\right| \geq & \left|E\left(P^{\prime}\right)\right|+\left|E\left(P_{1}\right)\right|+\left|E\left(P_{2}\right)\right|+2 \\
\geq & \frac{1}{2}\left[\left(\frac{\left|H_{0}\right|}{d}\right)^{r}+2+\sum\left(\frac{\left|A\left(L_{i}\right)\right|}{d}\right)^{r}+\sum \max \left\{1,\left|A\left(L_{i}\right)\right|-2\right\}\right. \\
& \left.+\left(\frac{(d-1) \sigma(\mathcal{M})}{d}\right)^{r}\right]+4 \\
> & \frac{1}{2}\left[\left(2+(b-1) n^{*} / d\right)^{r}+((d-1) \sigma(\mathcal{M}) / d)^{r}\right]+4 \quad(\text { by Lemma 3.1) } \\
> & \frac{1}{2}\left[2+n^{*}+(b-1)(d-1) \sigma(\mathcal{M}) / d\right]^{r}+4 \quad(\text { by Lemma 3.1) } \\
> & \frac{1}{2}(4(d-1) \sigma(\mathcal{M}))^{r}+3 \quad(\text { since } b \geq 4 d+1) \\
> & \frac{1}{2} n^{r}+3 \quad(\text { by }(5)) .
\end{aligned}
$$

As before, the desired cycle $C$ may be obtained by modifying $C^{*}$.

Now assume $z \in\left\{x_{0}, y_{0}\right\}$, and that the notation is chosen so that $z=x_{0}$. By Lemma 3.6(ii), there is a path $P_{2}$ in $\mathcal{M}-y_{0}$ from $x_{0}$ to some $y^{\prime \prime} \in N(y) \cap V\left(M_{m}\right)$ and containing no separating edge of $\mathcal{M}$ such that $\left|E\left(P_{2}\right)\right| \geq \frac{1}{2}\left(\sum\left\{\left(\frac{\left|A\left(M_{i}\right)\right|}{d}\right)^{r}: M_{i} \in\right.\right.$ $\left.\left.B_{1}(\mathcal{M})\right\}\right)+\left(\sum\left\{\max \left\{1,\left|A\left(M_{i}\right)\right|-2\right\}: M_{i} \in B_{2}(\mathcal{M})\right\}\right)+1$. By Lemma 3.5 there is a path $P_{1}$ in $L_{t+1} \ldots L_{\ell}$ from $c_{t}$ to $d_{t}$ such that $\left|E\left(P_{1}\right)\right| \geq \frac{1}{2}\left((d-1) n^{*} / d\right)^{r}$. Let $C^{*}:=\left(P^{\prime} \cup\left(Q-c_{t} d_{t}\right) \cup P_{1} \cup P_{2}\right)+\left\{y, y x, y y^{\prime \prime}\right\}$. Then by applying Lemma 3.1 as in the above paragraph (by swapping the roles of $L_{i}$ and $M_{i}$ ), we have

$$
\left|C^{*}\right| \geq \frac{1}{2}\left[(2+\sigma(\mathcal{M}))^{r}+\left((d-1) n^{*} / d\right)^{r}\right]+4 .
$$

If $(d-1) n^{*} / d \leq 2+\sigma(\mathcal{M})$, then by Lemma 3.1 and because $n^{*} \geq \sigma(\mathcal{M})-2$,

$$
\begin{aligned}
\left|C^{*}\right| & \geq \frac{1}{2}\left(2+\sigma(\mathcal{M})+(b-1)(d-1) n^{*} / d+2(b-1)\right)^{r}+3 \\
& >\frac{1}{2}(4(d-1) \sigma(\mathcal{M}))^{r}+3 \quad(\text { since } b \geq 4 d+1) \\
& >\frac{1}{2} n^{r}+3 \quad(\text { by }(5)) .
\end{aligned}
$$

So assume $(d-1) n^{*} / d \geq 2+\sigma(\mathcal{M})$. Applying Lemma 3.1 and (5) again, we have

$$
\left|C^{*}\right| \geq \frac{1}{2}\left((d-1) n^{*} / d+(b-1)(2+\sigma(\mathcal{M}))\right)^{r}+4>\frac{1}{2}(4 d \sigma(\mathcal{M}))^{r}+3>\frac{1}{2} n^{r}+3 .
$$

As before, the desired cycle $C$ can be obtained by modifying $C^{*}$.

Case 2. $\mathcal{M}$ may be chosen so that $x \notin\left\{x_{0}, y_{0}\right\} \cap\left\{c_{0}, d_{0}\right\},\left\{c_{0}, d_{0}\right\} \neq\left\{x_{0}, y_{0}\right\}$, and $\left\{x_{0}, y_{0}\right\} \subseteq V(\mathcal{H})$ 
We may assume that $\left\{c_{0}, d_{0}\right\} \subseteq V\left(H_{s}\right)$ and $\left\{c_{0}, d_{0}\right\} \neq\left\{a_{s-1}, b_{s-1}\right\}$, and $\left\{x_{0}, y_{0}\right\} \subseteq V\left(H_{t}\right)$ and $\left\{x_{0}, y_{0}\right\} \neq\left\{a_{t-1}, b_{t-1}\right\}$. Note that $a_{-1}$ and $b_{-1}$ are not defined. We consider only the case $s \leq t$, since the case $s \geq t$ is similar. By the choice of $\mathcal{L}$ and by $(5), \sigma(\mathcal{L}) \geq \sigma(\mathcal{M}) \geq \frac{n}{4(d-2)}$.

We claim that there is a path $P_{0}$ in $\mathcal{H}$ from $x$ to some $z \in\left\{c_{0}, d_{0}\right\} \cup\left\{x_{0}, y_{0}\right\}$ and containing no separating edge of $\mathcal{H}$ (except possibly $c_{0} d_{0}$ or $\left.x_{0} y_{0}\right)$ such that (a) $\left|E\left(P_{0}\right)\right| \geq \frac{1}{2}\left(\left|H_{0}\right| / d\right)^{r}+1$, (b) $c_{0} d_{0} \in E\left(P_{0}\right)$ or $x_{0} y_{0} \in E\left(P_{0}\right)$, (c) if $c_{0} d_{0} \in E\left(P_{0}\right)$, then $z \in\left\{x_{0}, y_{0}\right\}$ and $x_{0} y_{0} \notin E\left(P_{0}\right)$, and (d) if $x_{0} y_{0} \in E\left(P_{0}\right)$, then $z \in\left\{c_{0}, d_{0}\right\}$ and $c_{0} d_{0} \notin E\left(P_{0}\right)$.

Suppose $h=0$. We may assume $x \notin\left\{x_{0}, y_{0}\right\}$ (the case $x \notin\left\{c_{0}, d_{0}\right\}$ is symmetric). Let $H_{0}^{\prime}$ be obtained from $H_{0}$ by a T-transform at $\left\{x, x_{0} y_{0}\right\}$, and let $x^{\prime}$ denote the new vertex. By Theorem 1.2(b) we find a cycle $C_{0}$ in $H_{0}^{\prime}$ through $c_{0} d_{0}$ and $x x^{\prime}$ such that $\left|C_{0}\right| \geq \frac{1}{2}\left(\left|H_{0}\right| / d\right)^{r}+3$. Now $P_{0}:=C_{0}-x^{\prime}$ gives the desired path.

So we may assume $h \geq 1$. Let $H^{\prime}$ be obtained from $H_{1} \ldots H_{h}$ by deleting all separating edges of $\mathcal{H}$ different from $a_{0} b_{0}, c_{0} d_{0}$, and $x_{0} y_{0}$. Note that $H^{\prime}$ is 2-connected.

Assume $s=t=0$. Since $c_{0} d_{0} \neq x_{0} y_{0}$, we may assume $x_{0} y_{0} \neq a_{0} b_{0}$ (the case $c_{0} d_{0} \neq a_{0} b_{0}$ is the same). Suppose $c_{0} d_{0}=a_{0} b_{0}$. By Theorem 1.2(b) we find a cycle $C_{0}$ in $H_{0}$ through $a_{0} b_{0}$ and $x_{0} y_{0}$ such that $\left|C_{0}\right| \geq \frac{1}{2}\left(\left|H_{0}\right| / d\right)^{r}+3$. In $H^{\prime}-b_{0}$ we find a path $P^{\prime}$ from $x$ to $a_{0}$. Then $P_{0}:=\left(C_{0}-a_{0} b_{0}\right) \cup P^{\prime}$ gives the desired path. So assume $c_{0} d_{0} \neq a_{0} b_{0}$. Let $H_{0}^{\prime}$ be obtained from $H_{0}$ by an H-transform at $\left\{x_{0} y_{0}, a_{0} b_{0}\right\}$, and let $x^{\prime}, a^{\prime}$ denote the new vertices with $a^{\prime}$ subdividing $a_{0} b_{0}$. By Theorem 1.2(b), there is a cycle $C_{0}$ in $H_{0}^{\prime}$ through $c_{0} d_{0}$ and $a^{\prime} x^{\prime}$ such that $\left|C_{0}\right| \geq \frac{1}{2}\left(\left|H_{0}\right| / d\right)^{r}+3$. Let $P^{\prime}$ be a path in $H^{\prime}-a_{0} b_{0}$ from $x$ to the end, say $v$, of $C_{0}-\left\{a^{\prime}, x^{\prime}\right\}$ adjacent to $a^{\prime}$ and avoiding $\left\{a_{0}, b_{0}\right\}-\{v\}$. Then $P_{0}:=\left(C_{0}-\left\{a^{\prime}, x^{\prime}\right\}\right) \cup P^{\prime}$ gives the desired path.

Now assume $s=0<t$. Then $x_{0} y_{0} \neq a_{0} b_{0}$. By 2-connectivity of $H^{\prime}$, let $P^{\prime}$ be a path in $H^{\prime}-a_{0} b_{0}$ from $x$ to $z \in\left\{a_{0}, b_{0}\right\}$ through $x_{0} y_{0}$. By choosing appropriate notation, we may let $z=a_{0}$. Suppose $a_{0} b_{0}=c_{0} d_{0}$. By Theorem 1.2(c), we find a cycle $C_{0}$ in $H_{0}$ through $a_{0} b_{0}$ such that $\left|C_{0}\right| \geq \frac{1}{2}\left|H_{0}\right|^{r}+3$. Now $P_{0}:=\left(C_{0}-a_{0} b_{0}\right) \cup P^{\prime}$ gives the desired path. So we may assume $a_{0} b_{0} \neq c_{0} d_{0}$, and let $c_{0} \notin\left\{a_{0}, b_{0}\right\}$ (by choosing appropriate notation). If $d_{0} \in\left\{a_{0}, b_{0}\right\}$, then let $z^{\prime} \in\left\{a_{0}, b_{0}\right\}-\left\{d_{0}\right\}$, and apply Theorem 1.2(a) to find cycle $C_{0}$ in $\left(H_{0}+z^{\prime} c_{0}\right)-b_{0}$ through $a_{0} c_{0}$ such that $\left|C_{0}\right| \geq \frac{1}{2}\left(\left|H_{0}\right| / d\right)^{r}+2$; then $P_{0}:=\left(C_{0}-a_{0} c_{0}\right) \cup P^{\prime}$ gives the desired path. Now assume $d_{0} \notin\left\{a_{0}, b_{0}\right\}$. Let $H_{0}^{\prime}$ be obtained from $H_{0}$ by a T-transform at $\left\{a_{0}, c_{0} d_{0}\right\}$, and let $c^{\prime}$ denote the new vertex. We apply Theorem 1.2(a) to find a cycle $C_{0}$ in $\left(H_{0}^{\prime}+b_{0} c^{\prime}\right)-b_{0}$ through $a_{0} c^{\prime}$ such that $\left|C_{0}\right| \geq \frac{1}{2}\left(\left|H_{0}\right| / d\right)^{r}+2$. Now $P_{0}:=\left(C_{0}-c^{\prime}\right) \cup P^{\prime}$ gives the desired path.

Finally, we may assume $s \geq 1$. By exactly the same argument as for (1) of Case 2 in the proof of Lemma 4.2 , with $a_{0} b_{0}, c_{0} d_{0}, x_{0} y_{0}$ playing the roles of $f, p q, v w$, respectively, we find a path $P^{\prime}$ through $a_{0} b_{0}$ in $H^{\prime}$ from $x$ to $z \in\left\{c_{0}, d_{0}\right\} \cup\left\{x_{0}, y_{0}\right\}$ such that $P^{\prime}$ satisfies (b), (c), and (d). By Theorem 1.2(c), we find a cycle $C_{0}$ in $H_{0}$ through $a_{0} b_{0}$ such that $\left|C_{0}\right| \geq \frac{1}{2}\left|H_{0}\right|^{r}+3$. Now $P_{0}:=\left(C_{0}-a_{0} b_{0}\right) \cup\left(P^{\prime}-a_{0} b_{0}\right)$ gives the desired path.

Suppose $x_{0} y_{0} \in E\left(P_{0}\right)$. Without loss of generality, assume $z=c_{0}$. By Lemma 3.6(ii) there is a path $P_{1}$ in $\mathcal{L}-d_{0}$ from $c_{0}$ to some $y^{\prime} \in N(y) \cap V\left(L_{\ell}\right)$ and containing no separating edge of $\mathcal{L}$ such that $\left|E\left(P_{1}\right)\right| \geq \frac{1}{2}\left(\sum\left\{\left(\left|A\left(L_{i}\right)\right| / d\right)^{r}: L_{i} \in\right.\right.$ $\left.\left.B_{1}(\mathcal{L})\right\}\right)+\left(\sum\left\{\max \left\{1,\left|A\left(L_{i}\right)\right|-2\right\}: L_{i} \in B_{2}(\mathcal{L})\right\}\right)+1$. By Lemma 3.5, there is a path $P_{2}$ from $x_{0}$ to $y_{0}$ in $\mathcal{M}$ such that $\left|E\left(P_{2}\right)\right| \geq \frac{1}{2}((d-1) \sigma(\mathcal{M}) / d)^{r}+2$. Let $C^{*}$ be the cycle obtained from $\left(P_{0} \cup P_{1}\right)+\left\{y, y y^{\prime}, y x\right\}$ by replacing $x_{0} y_{0}$ with $P_{2}$. Then, as in Case 1 (with $\sigma(\mathcal{L})$ playing the role of $n^{*}$ ), by Lemma 3.1, and since $\sigma(\mathcal{L}) \geq \sigma(\mathcal{M})$, 
we have

$$
\begin{aligned}
\left|C^{*}\right| & \geq\left|E\left(P_{0}\right)\right|+\left|E\left(P_{1}\right)\right|+\left|E\left(P_{2}\right)\right|+1 \\
& >\frac{1}{2}\left[(2+\sigma(\mathcal{L}))^{r}+((d-1) \sigma(\mathcal{M}) / d)^{r}\right]+4 \\
& >\frac{1}{2}[(b-1)(d-1) \sigma(\mathcal{M}) / d]^{r}+3 \\
& >\frac{1}{2} n^{r}+3 \quad(\text { by }(5)) .
\end{aligned}
$$

Now assume $c_{0} d_{0} \in E\left(P_{0}\right)$. Without loss of generality, assume $z=x_{0}$. By Lemma 3.6(ii), there is a path $P_{1}$ from $x_{0}$ to some $y^{\prime} \in N(y) \cap V\left(M_{m}\right)$ in $\mathcal{M}-y_{0}$ such that $\left|E\left(P_{1}\right)\right| \geq \frac{1}{2}\left(\sum\left\{\left(\left|A\left(M_{i}\right)\right| / d\right)^{r}: M_{i} \in B_{1}(\mathcal{M})\right\}\right)+\left(\sum\left\{\max \left\{1,\left|A\left(M_{i}\right)\right|-2\right\}\right.\right.$ : $\left.\left.M_{i} \in B_{2}(\mathcal{M})\right\}\right)+1$. By Lemma 3.5, there is a path $P_{2}$ from $c_{0}$ to $d_{0}$ in $\mathcal{L}$ such that $\left|E\left(P_{2}\right)\right| \geq \frac{1}{2}\left(\frac{(d-1) \sigma(\mathcal{L})}{d}\right)^{r}+2$. Let $C^{*}$ be the cycle obtained from $\left(P_{0} \cup P_{1}\right)+\left\{y, y y^{\prime}, y x\right\}$ by replacing $c_{0} d_{0}$ with $P_{2}$. Then as in Case 1 and by Lemma 3.1,

$$
\begin{aligned}
\left|C^{*}\right| & \geq\left|E\left(P_{0}\right)\right|+\left|E\left(P_{1}\right)\right|+\left|E\left(P_{2}\right)\right|+1 \\
& \geq \frac{1}{2}\left[(2+\sigma(\mathcal{M}))^{r}+((d-1) \sigma(\mathcal{L}) / d)^{r}\right]+4 .
\end{aligned}
$$

If $(d-1) \sigma(\mathcal{L}) / d \geq 2+\sigma(\mathcal{M})$, then by Lemma 3.1 and by $(5)$,

$$
\left|C^{*}\right|>\frac{1}{2}((b-1) \sigma(\mathcal{M}))^{r}+4>\frac{1}{2}(4 d \sigma(\mathcal{M}))^{r}+3>\frac{1}{2} n^{r}+3 .
$$

Now assume $(d-1) \sigma(\mathcal{L}) / d \leq 2+\sigma(\mathcal{M})$. Then by Lemma 3.1 and $(5)$ and because $\sigma(\mathcal{L}) \geq \sigma(\mathcal{M})$

$$
\left|C^{*}\right|>\frac{1}{2}((b-1)(d-1) \sigma(\mathcal{L}) / d)^{r}+4>\frac{1}{2}(4(d-1) \sigma(\mathcal{M}))^{r}+3>\frac{1}{2} n^{r}+3 .
$$

As before, the desired cycle $C$ can be obtained by modifying $C^{*}$.

Case 3. For every choice of $\mathcal{M}$ with $\sigma(\mathcal{M}) \geq \frac{n}{4(d-2)}$, we have $x \in\left\{c_{0}, d_{0}\right\} \cap\left\{x_{0}, y_{0}\right\}$ or $\left\{c_{0}, d_{0}\right\}=\left\{x_{0}, y_{0}\right\}$.

Let $\mathcal{M}_{i}, 1 \leq i \leq k$, denote the $\mathcal{H} \mathcal{L}$-legs with $\sigma\left(\mathcal{M}_{i}\right) \geq \frac{n}{4(d-2)}$. Since $\sum\{\sigma(\mathcal{M})$ : $\mathcal{M}$ is an $\mathcal{H} \mathcal{L}$-leg as in Case 1 or Case 2$\} \leq \frac{(d-2-k) n}{4(d-2)}$ and because $n>(4 d+1)^{2}$, it follows from (3) and (4) that

$$
\sum_{i=1}^{k} \sigma\left(\mathcal{M}_{i}\right) \geq(n-1)-\frac{n-1}{4}-\frac{n-1}{4}-\frac{(d-2-k) n}{4(d-2)}-2 d>\frac{n}{4} .
$$

Let $G_{i}$ denote the graph obtained from $G$ by deleting all components of $(G-y)-$ $V\left(\mathcal{M}_{i} \cap(\mathcal{H} \cup \mathcal{L})\right)$ not containing any vertex of $\mathcal{M}_{i}$. Let $z \in\left\{c_{0}, d_{0}\right\}$ such that $z=x$ if $x \in\left\{c_{0}, d_{0}\right\}$. Since we are in Case $3, z \in V\left(\mathcal{M}_{i}\right)$ for $1 \leq i \leq k$. Let $t_{i}$ be the number of neighbors of $z$ in $G_{i}$ different from $y$ and not in $V\left(\mathcal{M}_{i} \cap(\mathcal{H} \cup \mathcal{L})\right)$. Then $t_{i} \geq 1$. We claim that $\sum_{i=1} t_{i} \leq d-1$. This is clear when $z=x$ because $y x$ is an edge of $G$. Now suppose $z \neq x$. Then $\left\{c_{0}, d_{0}\right\} \subseteq V\left(\mathcal{M}_{i}\right)$ for all $1 \leq i \leq k$. Since $z$ is incident with edges in both $\mathcal{H}-c_{0} d_{0}$ and $\mathcal{L}-c_{0} d_{0}$, we have $\sum_{i=1}^{k} t_{i} \leq d-1$.

Let $1 \leq s \leq k$ such that $\frac{\left|G_{s}\right|}{t_{s}}$ is maximum. Then $\frac{\left|G_{s}\right|}{t_{s}} \geq \frac{n}{4(d-1)}$. This follows from the following result (which can be proved by induction on $k$ ): If $\alpha_{1}+\cdots+\alpha_{k} \geq \alpha$ and $t_{1}+\cdots+t_{k}=m$, then $\max \left\{\frac{\alpha_{i}}{t_{i}}: 1 \leq i \leq k\right\} \geq \frac{\alpha}{m}$. 
For convenience, let $\left\{x_{s}, y_{s}\right\}=V\left(\mathcal{M}_{s} \cap(\mathcal{H} \cup \mathcal{L})\right)$, and assume, without loss of generality, $z=x_{s}=c_{0}$. Note that $G_{s}^{*}:=G_{s}+\left\{y x_{s}, y y_{s}, x_{s} y_{s}\right\}$ is 3-connected, $\Delta\left(G_{s}^{*}\right) \leq d+1$, and any vertex of degree $d+1$ must be incident with $x_{s} y$ or $x_{s} y_{s}$. By Theorem 1.2(a), there is a path $Q_{2}$ from $y_{s}$ to $y$ in $G_{s}^{*}-x_{s}$ such that

$$
\left|E\left(Q_{2}\right)\right| \geq \frac{1}{2}\left(\frac{(d-1)\left|G_{s}\right|}{d t_{s}}\right)^{r}+1 \geq \frac{1}{2}\left(\frac{n}{4 d}\right)^{r}+1 .
$$

Suppose $y_{s} \in V(\mathcal{H})$. By 2-connectivity of $\mathcal{H}$, there is a path $Q_{0}$ from $x$ to $y_{s}$ in $\mathcal{H}$ through $c_{0} d_{0}$. By Lemma 3.5, there is a path $Q_{1}$ from $c_{0}$ to $d_{0}$ in $\mathcal{L}$ such that $\left|E\left(Q_{1}\right)\right| \geq \frac{1}{2}\left(\frac{(d-1) \sigma(\mathcal{L})}{d}\right)^{r}+2$. Let $C^{*}:=\left(\left(Q_{0}-c_{0} d_{0}\right) \cup Q_{1} \cup Q_{2}\right)+y x$. Then

$$
\left|C^{*}\right| \geq\left|E\left(Q_{1}\right)\right|+\left|E\left(Q_{2}\right)\right| \geq \frac{1}{2}\left[\left(\frac{(d-1) \sigma(\mathcal{L})}{d}\right)^{r}+\left(\frac{n}{4 d}\right)^{r}\right]+3 .
$$

If $\frac{(d-1) \sigma(\mathcal{L})}{d} \geq \frac{n}{4 d}$, then by Lemma 3.1 and since $b \geq 4 d+1$,

$$
\left|C^{*}\right| \geq \frac{1}{2}\left(\frac{(b-1) n}{4 d}\right)^{r}+3 \geq \frac{1}{2} n^{r}+3 .
$$

Now assume $\frac{(d-1) \sigma(\mathcal{L})}{d} \leq \frac{n}{4 d}$. By Lemma 3.1 and since $\sigma(\mathcal{L}) \geq \sigma(\mathcal{M})>\frac{n}{4(d-2)}$ (by $(5))$,

$$
\left|C^{*}\right| \geq \frac{1}{2}\left[\frac{(b-1)(d-1) \sigma(\mathcal{L})}{d}\right]^{r}+3>\frac{1}{2} n^{r}+3 .
$$

As before, the desired cycle $C$ can be obtained by modifying $C^{*}$.

Thus, we may assume $y_{s} \notin V(\mathcal{H})$. Then $z=x$ and $y_{s} \in V\left(L_{t}\right)$ for some $1 \leq t<\ell$ $(t \neq \ell$ by the choice of $\mathcal{L})$. Let $n^{*}:=\sum_{i=t+1}^{\ell}\left|A\left(L_{i}\right)\right|$. Note that $n^{*} \leq \sigma\left(L_{t+1} \ldots L_{\ell}\right)$. By our choice of $\mathcal{L}, n^{*} \geq \sigma(\mathcal{M})-2$. By 2-connectivity, let $Q_{0}$ be a path from $x$ to $y_{s}$ through $c_{t} d_{t}$ in $L_{1} \ldots L_{t}$. Note that $\left|E\left(Q_{0}\right)\right| \geq 2$. By Lemma 3.5 there is a path $Q_{1}$ from $c_{t}$ to $d_{t}$ in $L_{t+1} \ldots L_{\ell}$ such that $\left|E\left(Q_{1}\right)\right| \geq \frac{1}{2}\left(\frac{(d-1) n^{*}}{d}\right)^{r}+1$. Let $C^{*}:=\left(\left(Q_{0}-c_{t} d_{t}\right) \cup Q_{1} \cup Q_{2}\right)+y x$. Then

$$
\left|C^{*}\right| \geq\left|E\left(Q_{1}\right)\right|+\left|E\left(Q_{2}\right)\right|+2 \geq \frac{1}{2}\left[\left(\frac{(d-1) n^{*}}{d}\right)^{r}+2+\left(\frac{n}{4 d}\right)^{r}\right]+3 .
$$

If $\frac{(d-1) n^{*}}{d} \geq \frac{n}{4 d}$, then by Lemma 3.1 and since $b \geq 4 d+1$,

$$
\left|C^{*}\right| \geq \frac{1}{2}\left(\frac{(b-1) n}{4 d}\right)^{r}+3 \geq \frac{1}{2} n^{r}+3 .
$$

Now assume $\frac{(d-1) n^{*}}{d} \leq \frac{n}{4 d}$. Then by Lemma 3.1 and since $n^{*} \geq \sigma(\mathcal{M})-2>\frac{n}{4(d-1)}-2$ (by (5)),

$\left|C^{*}\right|>\frac{1}{2}\left(\frac{(b-1)(d-1) n^{*}}{d}+2(b-1)\right)^{r}+3>\frac{1}{2}\left[4(d-1) n^{*}+2(b-1)\right]^{r}+3>\frac{1}{2} n^{r}+3$.

Again, the desired cycle $C$ can be obtained by modifying $C^{*}$. 
6. Conclusions. We now complete the proof of Theorem 1.2.

Proof of Theorem 1.2. Let $n, d, r, G$ be given as in Theorem 1.2. We apply induction on $n$. When $n=5, G$ is isomorphic to one of the following three graphs: $K_{5}, K_{5}$ minus an edge, or the wheel on five vertices. In each case, we can verify that Theorem 1.2 holds. So assume that $n \geq 6$ and Theorem 1.2 holds for all 3 -connected graphs with at most $n-1$ vertices. Then Theorem 1.2(a) holds by Lemma 4.1, Theorem 1.2(b) holds by Lemma 4.2, and Theorem 1.2(c) holds by Lemma 5.1. This completes the proof of Theorem 1.2.

Our proof of Theorem 1.2 implies a polynomial time algorithm which, given a 3 -connected $n$-vertex graph, finds a cycle of length $\frac{1}{2} n^{r}+3$. When combined with the next two results [8], our proof implies a cubic algorithm.

Lemma 6.1. Let $G$ be a $k$-connected graph where $k$ is a positive integer. Then $G$ contains a k-connected spanning subgraph with $O(V)$ edges; such a subgraph can be found in $O(E)$ time.

Lemma 6.2. Let $G$ be a 2-connected graph and let e, $f \in E(G)$. Then there is a cycle through e and $f$ in $G$, and such a cycle can be found in $O(V)$ time.

Lemma 6.2 is actually an easy consequence of a result in [8] which states that, in a 2-connected graph $G$, one can find, in $O(V)$ time, two disjoint paths linking two given vertices. Our algorithm is similar to that in [3]. Therefore, we give only an outline and omit complexity analysis.

Algorithm. Let $G$ be a 3 -connected graph with $\Delta(G) \leq d$, and assume $|G| \geq 5$. The following procedure finds a cycle $C$ in $G$ with $|C| \geq \frac{1}{2}|G|^{r}+3$.

1. Preprocessing. Replace $G$ with a 3 -connected spanning subgraph of $G$ with $O(|G|)$ edges.

2. We either find the desired cycle $C$, or we reduce the problem to Theorem 1.2 for some 3-connected graphs $G_{i}$, for which $\left|G_{i}\right|<|G|$ and each $G_{i}$ contains a vertex which does not belong to any other $G_{i}$.

3. Replace each $G_{i}$ with a 3-connected spanning subgraph of $G_{i}$ with $O\left(\left|G_{i}\right|\right)$ edges.

4. Apply Lemma 4.1 to those $G_{i}$ for which Theorem 1.2(a) needs to be applied. Apply Lemma 4.2 to those $G_{i}$ for which Theorem 1.2(b) needs to be applied. Apply Lemma 5.1 to those $G_{i}$ for which Theorem 1.2(c) needs to be applied.

5 . Repeat steps 3 and 4 for new 3 -connected graphs.

6 . In the final output, replace all virtual edges by appropriate paths in $G$ to complete the desired cycle $C$.

Acknowledgment. We thank the referees for their suggestions that helped improve the presentation of this paper.

\section{REFERENCES}

[1] A. BJöRKLund And T. Husfeldt, Finding a path of superlogarithmic length, SIAM J. Comput., 32 (2003), pp. 1395-1402.

[2] G. Chen, Z. GaO, X. Yu, And W. Zang, Approximating the longest cycle problem on graphs with bounded degree, Lecture Notes in Comput. Sci. 3595, 2005, pp. 870-884.

[3] G. Chen, J. Xu, And X. Yu, Circumference of graphs with bounded degree, SIAM J. Comput., 33 (2004), pp. 1136-1170.

[4] T. Feder, R. Motwani, And C. Subi, Approximating the longest cycle problem in sparse graphs, SIAM J. Comput., 31 (2002), pp. 1596-1607.

[5] T. Feder And R. Motwani, Finding a long cycle in a graph with a degree bound and a 3cyclable minor, manuscript, 2004. 
[6] H. N. Gabow, Finding paths and cycles of superpolylogarithmic length, in Proceedings of the 36th Annual ACM Symposium on Theory of Computing (STOC), 2004, pp. 407-416.

[7] J. E. Hopcroft And R. E. TARJAn, Dividing a graph into triconnected components, SIAM J. Comput., 2 (1973), pp. 135-158.

[8] T. Ibaraki and H. Nagamochi, A linear-time algorithm for finding a sparse $k$-connected spanning subgraph of a k-connected graph, Algorithmica, 7 (1992), pp. 583-596.

[9] B. JaCKSON AND N. C. Wormald, Longest cycles in 3-connected graphs of bounded maximum degree, in Graphs, Matrices, and Designs, R. S. Rees, ed., Dekker, New York, 1993, pp. 237254.

[10] D. Karger, R. Motwani, And G. D. S. Ramkumar, On approximating the longest path in a graph, Algorithmica, 18 (1997), pp. 82-98.

[11] W. T. TutTe, Connectivity in Graphs, University of Toronto Press, Toronto, 1966. 